\title{
Service Station Evaluation Problem in Catering Service of High-Speed Railway: A Fuzzy QFD Approach Based on Evidence Theory
}

\author{
Xin $W u,{ }^{1,2}$ Lei Nie, ${ }^{1,2}$ and Meng $X u^{2}$ \\ ${ }^{1}$ School of Traffic and Transportation, Beijing Jiaotong University, Beijing 100044, China \\ ${ }^{2}$ State Key Laboratory of Rail Traffic Control and Safety, Beijing Jiaotong University, Beijing 100044, China \\ Correspondence should be addressed to Lei Nie; lnie@bjtu.edu.cn
}

Received 10 April 2015; Revised 17 June 2015; Accepted 24 June 2015

Academic Editor: Zdeněk Kala

Copyright (C) 2015 Xin Wu et al. This is an open access article distributed under the Creative Commons Attribution License, which permits unrestricted use, distribution, and reproduction in any medium, provided the original work is properly cited.

Catering Service of High-Speed Railway (CSHR) starts at suppliers, includes distribution centers and service stations in cities, and ends at cabinets in high-speed trains. In Distribution System Design (DSD) Problem for CSHR, it is critical to evaluate the alternatives of service stations, which is termed as Service Station Evaluation Problem in Catering Service of High-speed Railway (SSEP-CSHR). As a preparation work for DSD, SSEP-CSHR needs to be solved without detailed information and being accompanied with uncertainty. Fuzzy Quality Function Deployment (F-QFD) has been given in the literatures to deal with vagueness in Facility Location Evaluation (FLE). However, SSEP-CSHR that includes identifying and evaluating stations requires not only dealing with the vague nature of assessments but also confirming them. Based on evidence theory, this paper introduces the framework to give the truth of proposition " $x$ is A." Then it is incorporated into a two-phase F-QFD with an approximate reasoning to enable the truth of the decisions to be measured. A case study that refers to 85 alternative stations on Chinese high-speed railway will be carried out to verify the proposed method. Analysis shows that the proposed evaluation method enhances scientific credibility of FLE and allows decision makers to express how much is known.

\section{Introduction}

In China, the significance of Catering Service of High-Speed Railway (CSHR) has emerged with the rapid development of high-speed passenger rail line's construction. The process of the service starts at suppliers, includes distribution centers and service stations in various cities, and ends at cabinets in high-speed trains (see Figure 1). Service stations, that is, places to catering foods, play an essential role as link-ups between suppliers and trains in food distribution system. The performance of each station for catering is extremely important for CSHR to maintain a continuous supply and rapid distribution. Hence for a new institute to establish a distribution system to support CSHR, it is an inevitable decision to evaluate alternative sites of service station as a preliminary work for other further decisions in CSHR. Service station Evaluation Problem (SSEP-CSHR) thus becomes a critical issue in CSHR.
The evaluation of alternative locations is a Multi-Criteria Decision-Making (MCDM) problem referring to qualitative criteria. Under this situation, the values referred in MCDM are often imprecisely defined for the decision-makers. So a fuzzy perspective is adopted naturally in related literatures to deal with above uncertainty. Kuo et al. [1] develop a decision support system in basis of Fuzzy Analytic Hierarchy Process (F-AHP) to assess new stores. Chen [2] proposes a MCDM approach to solve a distribution center evaluation problem under fuzzy environment. Kahraman et al. [3] solve Facility Location Evaluation (FLE) problem by four different fuzzy group decision-making methods, including a fuzzy model proposed by Blin [4], fuzzy synthetic evaluation, weighted goals method [5], and Extent Analysis by Chang [6]. Kulak et al. [7] develop an MCDM based on axiomatic design and Analytic Hierarchy Process (AHP) to select transportation companies. Guo [8] proposes a Fuzzy Data Envelopment Analysis (F-DEA) model for a case involving a new restaurant 


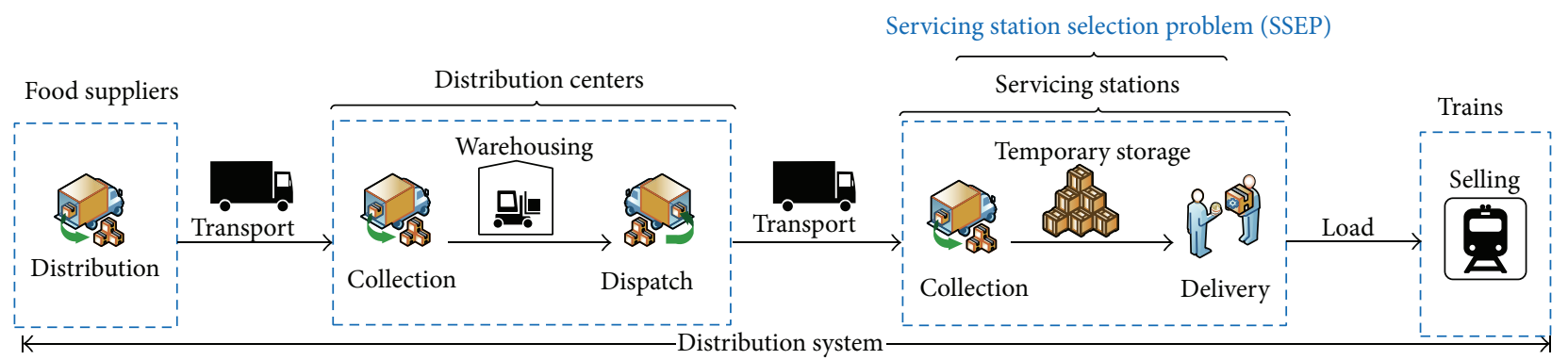

FIgURE 1: The process of catering service for High-Speed Railway (CSHR).

location. Like other MCDM problems, SSEP-CSHR is also required to be done without detailed information and thus is always accompanied with different types of uncertainty.

Service stations in CSHR are also required to satisfy some Basic Requirements, for example, accessibility to trains and, meanwhile, enough time for catering food. Quality Function Deployment (QFD) is one of coherent systems widely used to translate Basic Requirements into actionable alternatives [9]. A typical QFD contains info about "WHATs" and "HOWs," the relations between "WHATs" and "HOWs," and the correlations between "WHATs"/"HOWs" themselves. All above information can be presented by Houses of Quality (HOQ) [10].

Jamalnia et al. [11] have attempted to develop a Fuzzy Quality Function Deployment (F-QFD) approach for FLE. they proposed some facility locations requirements and then use alternative location to realize them. It follows that FQFD methodology is capable of solving SSEP-CSHR as a special case of FLE problems. But one of challenges that FLE-F-QFD is encountering when it is utilized in SSEPCSHR is accountability. Because expensive cost will be spent if a decision-maker plans to establish a cold chain for CSHR and SSEP-CSHR that involves identifying, evaluating alternative service stations requires not only handling the vague nature of assessments but also confirming them, that is, professing what decision makers do know and what they do not know [12]; It means that decision makers lead the truth of the outputs to be measured. Furthermore CSHR is a new field in logistic management, experts are far from having general agreement about the importance of certain Basic Requirements. So the results provided by FLE-F-QFD are indispensable to be crosschecked through various experts to ensure its credibility.

It leads to some important problems in related studies about uncertainty management in F-QFD. Kim et al. [13] conclude the uncertainty in QFD mainly arising from the following sources, Fuzziness, Fluctuation, and Heterogeneity.

(1) Fuzziness accounts for the imprecise nature or vagueness of the linguistic terms used in QFD. In fact, QFD teams usually do not have sufficient knowledge about the values taken in a HOQ due to the lack of clarity. It makes the application of fuzzy sets theory in QFD (i.e., F-QFD) significant in many problems; fuzzy sets are used to describe natural language-based criteria. F-QFD is one of extended version of typical QFD approaches, which have been widely used in many field [11, 14-20]. In F-QFD, (Fuzzy) AHP approaches $[6,21-25]$ are always used to generate the inputs.

(2) Fluctuation is associated with the change of Basic Requirements over time. SSEP-CSHR is placed on the early stage of SCD problem, so the Fluctuation of the requirements is inevitable. The framework of Robust QFD was presented by Kim et al. $[13,26]$ to accommodate this uncertainty and to prevent QFD teams obtaining misleading outputs.

(3) Heterogeneity comes from the different viewpoints of multiple experts. Hence it is required to justify the outputs obtained by a QFD team by other experts' opinions.

To achieve justifications to cope with the Heterogeneity, evidences from questionnaires must be referred to. Evidences from questionnaires are generally related to the occurrence of deterministic or stochastic events and treat crisp values or probability distributions as their paradigm. However sometimes the evidences involve nonrandom events, such as "an estimated strength of one passenger demand," and thus are biased by perception or even guess [12]. SSEPCSHR apparently refers to subjective experience or uncertain considerations. In this case, evidences are represented by possibility distributions, or mixed evidences consist of both probability and possibility distribution. In regard to the above-mentioned status, FLE-F-QFD should be improved to adapt above complicated evidences in order to solve SSEPCSHR.

Recently, great advances have been made in the field of evidence theory. Then complicated empirical evidence is utilized to confirm propositions " $x$ is $\mathbf{A}$ " in evidence theory. Various types of proofs are thus defined by the way of an evidence $x$ proving its membership in any set $\mathbf{A}$ [27]. The objective of this paper is to introduce evidence theory to make an approximating reasoning possible in a modified QFD to dampen the effects of both Fuzziness and Heterogeneity.

Figure 2 concludes the relations among application background, motivations, existing versions of QFD, and our proposed method. In the paper, we firstly present the essence of evidence theory including its concepts and formulas to measure the truth of " $x$ is A." Meanwhile we propose two measures which will be used in our modified F-DFD. Further two-phase F-QFD is introduced. Based on the two-phase FQFD method, we devote ourselves to incorporate evidence theory into SSEP-CSHR by a procedure of approximate reasoning and form an effective approach to determine alternatives in CSHR with respect to certain qualitative criteria. 


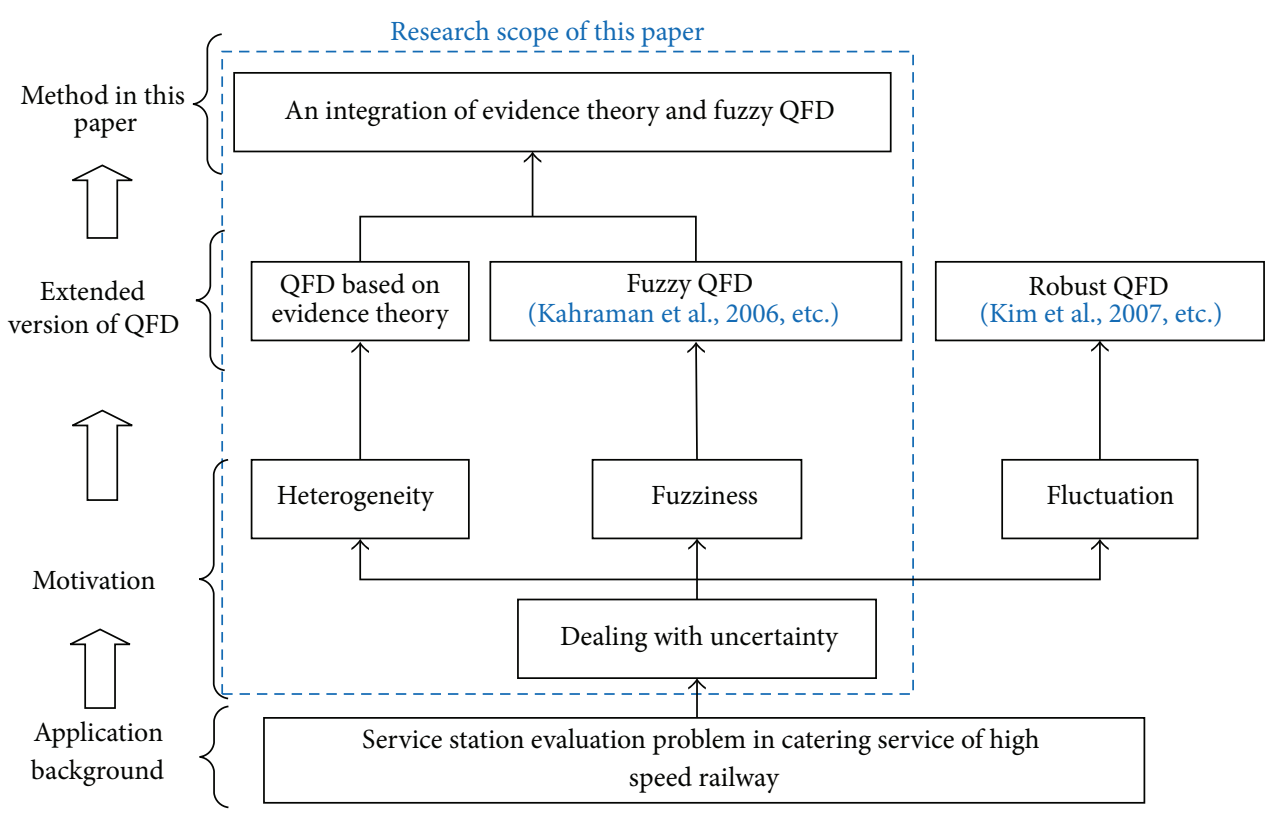

FIGURE 2: The precedence relationship among background, motivations, various QFDs, and our method.

This paper is organized as follows. In Section 2, a framework for determining the truth of " $x$ is $\mathbf{A}$ " in basis of evidence theory is introduced. In Section 3.2, a two-phase FQFD methodology will be described briefly and in Section 3.2 HOQs in the context of SSEP-CSHR will be discussed in detail. Further, in Section 3.3, a procedure of approximate reasoning based on evidence theory is incorporated into FLEF-QFD for SSEP-CSHR. In Section 4, a case study in the context of CSHR will be done to verify our methodology. The analysis shows that the proposed method enhances scientific credibility of FLE-F-QFD in CSHR and allows decision makers to express how much is known. Figure 3 illustrates the precedence relationship between our sections.

\section{Framework for Determining the Truth of " $x$ Is A" Based on Evidence Theory}

2.1. Proposition. The canonical form of a proposition is " $x$ is A," where subject $x$ refers to the condition of an object and $\mathbf{A}$ is a set that represents a predicate of $x$. The following are examples [12].

Example 1. Constructions Costs (CC) are very important.

$$
x=\text { Constructions Costs (CC); } \mathbf{A}=\text { very important. }
$$

Example 2. Proximity to trains (PT) is quite important.

$$
x=\text { proximity to trains }(\mathrm{PT}) ; \mathbf{A}=\text { quite important. }
$$

A is represented by fuzzy sets. Truth of a proposition is evaluated by the degree of $x$ supporting $\mathbf{A}$.

2.2. Set $\mathbf{A}$ in " $x$ Is $\mathbf{A}$ ". The predicate $\mathbf{A}$ is a description of a situation and is treated as either crisp or fuzzy sets.
Given $\widetilde{\mathbf{A}}=\{(x, \widetilde{\mathbf{A}}(x)) \mid x \in \mathbf{U}\}$ is a fuzzy set, where $\widetilde{\mathbf{A}}(x)$ denotes normalized membership function, $\mathbf{U}$ is a universal set. According to the first decomposition theorem:

$$
\widetilde{\mathbf{A}}=\bigcup_{\alpha \in[0,1]} \alpha \cdot \mathbf{A}_{\alpha} .
$$

And sets called $\alpha$-cut of the fuzzy set are defined as follows:

$$
\mathbf{A}_{\alpha}=\{x \in \mathbf{U} \mid \widetilde{\mathbf{A}}(x) \geq \alpha\}=\left[(A)_{\alpha}^{L},(A)_{\alpha}^{U}\right]
$$

$$
\forall \alpha \in[0,1] .
$$

We set $\alpha \in\{1 / K, 2 / K, \ldots, 1\} . K$ is the number of $\alpha$ cut considered in $[0,1]$. For brevity, in this paper, we use Triangular Fuzzy Numbers (TFNs) $a / b / c$ to denote a fuzzy number on an $x$ - $\alpha$ graph; say $\alpha=1$ with $x=b$, and say $\alpha=0$ with $x$ in $[a, c]$ [27].

A membership function is a graphical expression about decision makers' opinions. For example, a group of decision makers is asked to express "the strength degree of the relation between CP and PT" by fuzzy scores. Each decision maker draws two lines on an $x-y$ graph to describe the feeling about the strength, $x$-axis being the score, and $y$-axis being the membership level between 0 and 1 (like a triangle or a trapezoid). As shown in Figure 4(a), two lines will both start from $y=1$ at $x=5$, which means that "the strength of the relation between CP and PT is 5 " is with the most certainty, and end at two points, respectively, on left and right side of score 5 , where the scores are totally uncertain for the decision maker (thus $y=0$ at the points). Many similar lines are drawn by different decision makers and a membership function can be obtained by combining them as shown in Figure 4(a). In Figure 4(b), we take $K=5$; $\alpha$-cuts of the fuzzy set are used to fit the depicted membership function. The larger the parameter $K$ is, the better the $\alpha$-cuts fit the function. 


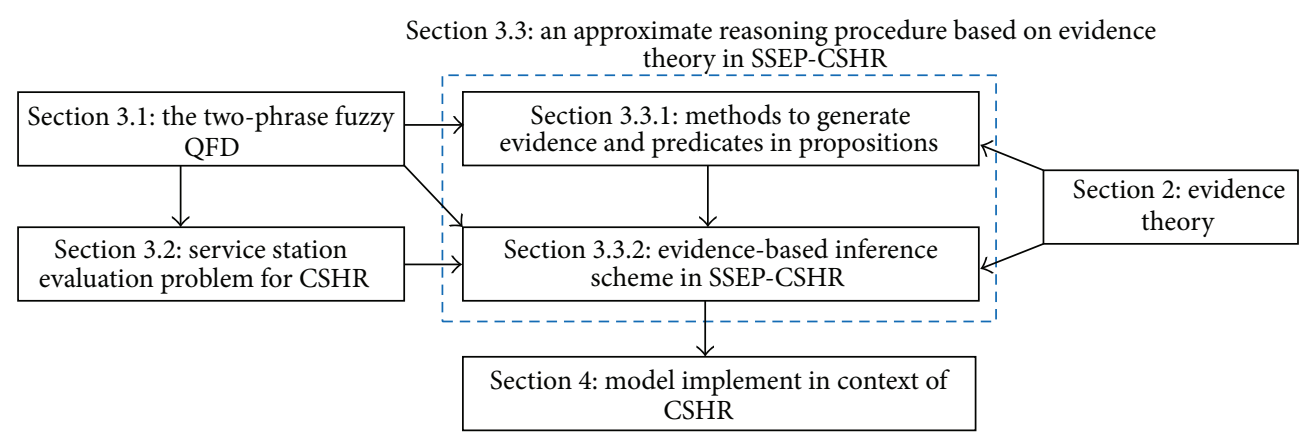

FIgURE 3: The precedence relationship between sections.

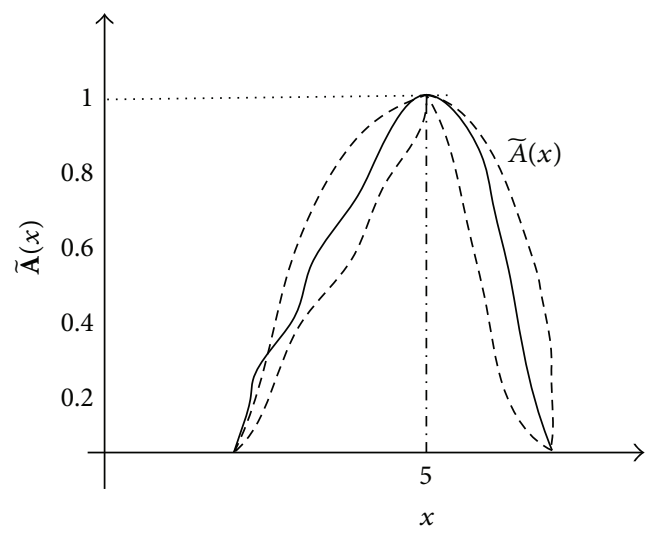

(a)

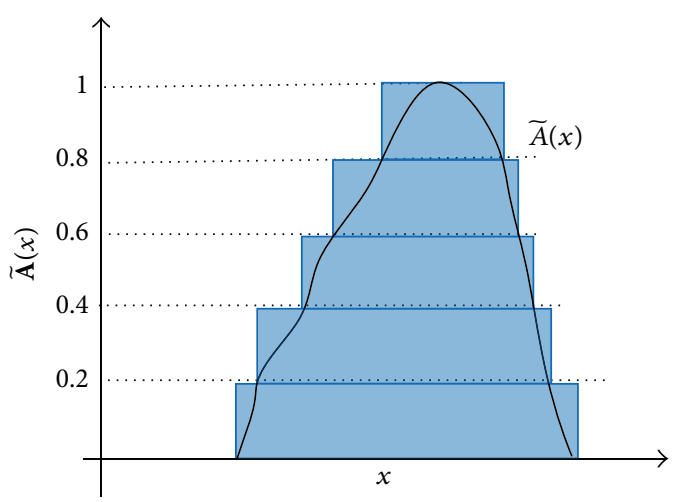

(b)

FIgURE 4: An illustration of the development of predicate $A$.

2.3. Evidence about $x$. Let a universal set be $\mathbf{U}$. The family of all subsets of a given set $\mathbf{A}$ is called the power set of $\mathbf{A}$, and it is denoted by $\mathscr{P}(\mathbf{A})$. The following function is called basic probability assignment of evidences:

$$
m: \mathscr{P}(\mathbf{U}) \longrightarrow[0,1]
$$

such that $m(\varnothing)=0$ and

$$
\sum_{A \in \mathscr{P}(\mathbf{U})} m(\mathbf{A})=1
$$

Every set $\mathbf{A}$ in $\mathscr{P}(\mathbf{U})$ for which $m(\mathbf{A})>0$ is usually named a focal element of $m$ that is a subset of $\mathbf{U}$ on which the available evidence focuses. The pair $(\mathbf{F}, m)$ is termed as a body of evidence (BOE), where $\mathbf{F}$ denotes a set of focal elements and $m$ denotes the associated basic assignment. Moreover, because $m$ can be fully characterized by a list of focal elements $\mathbf{F}=\left\{\mathbf{f}_{1}, \mathbf{f}_{2}, \ldots, \mathbf{f}_{M}\right\}$; therefore in the paper $\mathbf{F}$ is used instead of $(\mathbf{F}, m)$ for short [27].

According to the properties of a BOE, the evidence about $x$ is divided into three types.

(1) Conflicting Evidence (C-BOE). The BOE represents a random situation that each focal element is conflicting with one another, that is, for each $i \neq j \in\{1,2, \ldots, M\}$ :

$$
\mathbf{f}_{i} \cap \mathbf{f}_{j}=\varnothing \text {. }
$$

Each event yields a piece of evidence pointing to distinct focal elements. Based on the frequency at which pieces of evidence focuse on each focal element, a probability distribution is developed.

(2) Nested Evidence (N-BOE). In this type of BOE, pieces of evidence point to a range of nested sets. Nested sets indicate the focal elements in $\mathbf{F}$ which can be sorted as follows:

$$
\mathbf{f}_{(1)} \subseteq \mathbf{f}_{(2)} \cdots \subseteq \mathbf{f}_{(M)} .
$$

This is a situation that one faces in the data that describes estimated quantities. The distribution of this type is termed the possibility distribution.

(3) Mixed Evidence (M-BOE). This type is a generalization of $\mathrm{C}-\mathrm{BOE}$ and N-BOE. In this case, some pieces of evidence point to sets that are nested and some to mutually exclusive sets.

By Venn Diagram, above types of BOEs are illustrated in Figure 5. Figure 5(a) is a C-BOE. Figure 5(b) is a N-BOE. Figure 5 (c) is a M-BOE. All the BOEs satisfy $m\left(\mathbf{f}_{1}\right)+m\left(\mathbf{f}_{2}\right)+$ $m\left(\mathbf{f}_{3}\right)+m\left(\mathbf{f}_{4}\right)+m\left(\mathbf{f}_{5}\right)+m\left(\mathbf{f}_{6}\right)=1$.

A further question is how to develop the BOEs in SSEP-CSHR context. For example, a statement proposed by decision makers is "Cost performance (CP) is important." How do we generate a BOE to support the statement? 


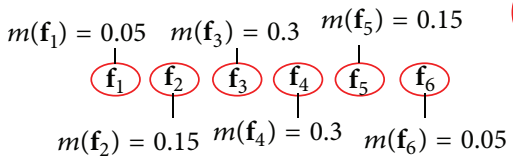

(a) C-BOE
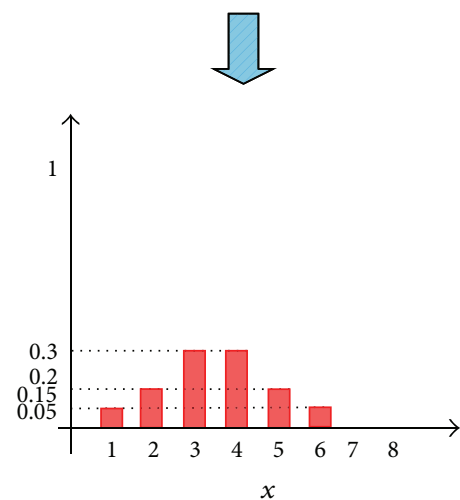

(d) C-BOE

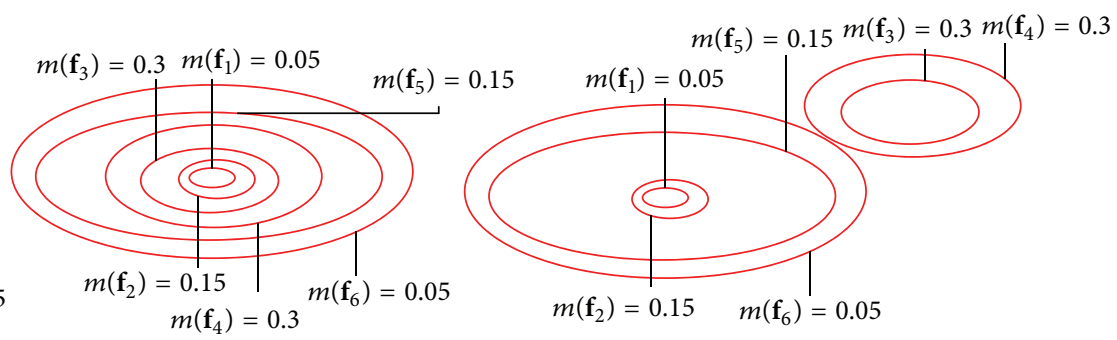

(b) N-BOE
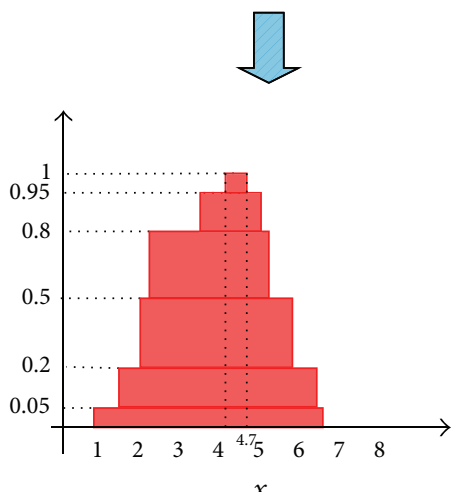

(e) N-BOE (c) $\mathrm{M}-\mathrm{BOE}$
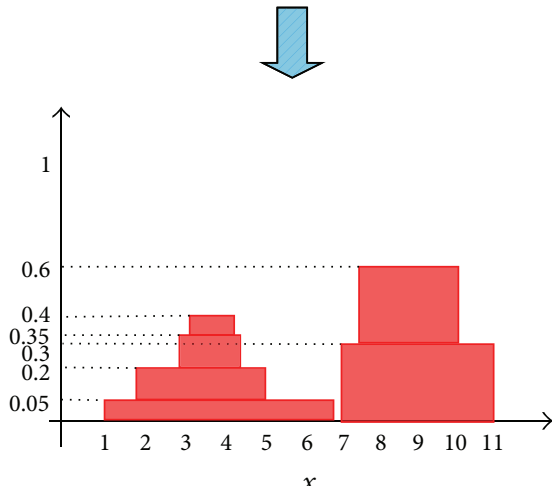

(f) $\mathrm{M}-\mathrm{BOE}$

FIGURE 5: Three illustrative examples of body of evidence (BOE).

A C-BOE is often developed by choice questions with mutually exclusive options in questionnaires. Each interviewee is asked to select only one option from options. The accumulative frequency of each option gives a probability distribution. For example, in Figure 5(d) each interviewee is asked to select a score from the set $\{1,2,3,4,5,6,7,8\}$.

An approach to develop N-BOE or M-BOE is to ask each interviewee to indicate the range of her agreeable score, and the enquirer draws a horizontal line corresponding to the range, and finally stacks the responses. If $M$ people are asked during our survey, the height of one horizontal line is $1 / M$. If a representation of the set of opinions is in general agreement, the evidence can be viewed as a possibility distribution (i.e., N-BOE) [12]. The differences among the opinions are only boundaries of the agreeable ranges. For instance, in Figure 5(e), we assume an "ideal" score ranging from 4 to 4.7 exists, which every interviewee agrees on, and interviewees give their acceptable closeness to the "ideal" score range. Sometimes evidences from questionnaires are without any general agreement. Then the evidence is an $\mathrm{M}$ BOE. As shown in Figure 5(f), M-BOE needs no requirements of an "ideal" score range, so M-BOE can be used to describe the opinions without any consensus. Owing to info deficiency during solving SSEP-CSHR, sometimes SSEPCSHR is required to deal with $\mathrm{M}-\mathrm{BOE}$.

2.4. Measuring the Truth of " $x$ Is A". How BOE and set A are combined dictates the framework for determining the truth of " $x$ is $\mathbf{A . "}$

Given a fuzzy set $\widetilde{\mathbf{A}}(x)$ and a $\mathrm{BOE}(\mathbf{F}, m)$ on $\mathbf{U}$, where $\mathbf{F}=$ $\left\{\mathbf{f}_{1}, \ldots, \mathbf{f}_{M}\right\}$, a framework to determine the truth of " $x$ is $\mathbf{A}$ " is based on two dual measures: Belief (Bel) and Plausibility (Pl) as follows:

$$
\begin{aligned}
\operatorname{Bel}(\mathbf{F}, \widetilde{\mathbf{A}}) & =\frac{1}{K} \sum_{i=1}^{M} m\left(\mathbf{f}_{i}\right) \sum_{\alpha \in[0,1]} I_{\left\{\mathbf{f}_{i} \subset\left[(A)_{\alpha}^{L},(A)_{\alpha}^{U}\right]\right\}}, \\
\operatorname{Pl}(\mathbf{F}, \widetilde{\mathbf{A}}) & =\frac{1}{K} \sum_{i=1}^{M} m\left(\mathbf{f}_{i}\right) \sum_{\alpha \in[0,1]} I_{\left\{\mathbf{f}_{i} \cap\left[(A)_{\alpha}^{L},(A)_{\alpha}^{U}\right] \neq \varnothing\right\}}
\end{aligned}
$$

such that if $\widetilde{\mathbf{A}}=\varnothing$, then $\operatorname{Bel}(\mathbf{F}, \widetilde{\mathbf{A}})=\operatorname{Pl}(\mathbf{F}, \widetilde{\mathbf{A}})=0$ and if $\widetilde{\mathbf{A}}=\mathbf{U}, \operatorname{Bel}(\mathbf{F}, \widetilde{\mathbf{A}})=\operatorname{Pl}(\mathbf{F}, \widetilde{\mathbf{A}})=1$. In addition,

$$
\begin{aligned}
\operatorname{Bel}(\mathbf{F}, \widetilde{\mathbf{A}}) & =1-\operatorname{Pl}(\mathbf{F}, \overline{\widetilde{\mathbf{A}}}) ; \\
\operatorname{Pl}(\mathbf{F}, \widetilde{\mathbf{A}}) & =1-\operatorname{Bel}(\mathbf{F}, \overline{\widetilde{\mathbf{A}}}) .
\end{aligned}
$$

In (7) and (8), $K$ is the number of $\alpha$-cuts considered in $[0,1]$; $I_{\{p\}}$ is a Boolean function.

While, for every $i \in\{1,2, \ldots, M\}, m\left(\mathbf{f}_{i}\right)$ characterizes the frequency of pieces of evidence focusing on exactly focal elements $\mathbf{f}_{i}$ alone, equation (7) represents the total evidence that each focal element belongs to every $\alpha$-cut of the fuzzy set. Equation (8) represents not only the evidence that each focal element belongs to every $\alpha$-cut, but also the additional evidence associating with the focal element overlapping every $\alpha$-cut. Hence, the Bel is a conservative measure and the $\mathrm{Pl}$ is an optimistic one. Then the truth of " $x$ is $\mathbf{A}$ " can be defined by following weighted summation:

$$
T(\mathbf{F}, \widetilde{\mathbf{A}})=w_{1} \operatorname{Bel}(\mathbf{F}, \widetilde{\mathbf{A}})+w_{2} \operatorname{Pl}(\mathbf{F}, \widetilde{\mathbf{A}}) .
$$


Based on the above discussion, the types of BOE are divided into $\mathrm{C}-\mathrm{BOE}, \mathrm{N}-\mathrm{BOE}$, and $\mathrm{M}-\mathrm{BOE}$. The types of set for $\mathbf{A}$ that we are mainly concerned with are the crisp set and the fuzzy set. Hence there are six cases of the proposition, " $x$ is A," corresponding to the combination of the type of BOE and the type of set A. We give an illustrative example to illustrate the two measures.

Let $w_{1}=w_{2}=0.5$ and $K=5$. Let $m\left(\mathbf{f}_{1}\right)=0.05 ; m\left(\mathbf{f}_{2}\right)=$ $0.15 ; m\left(\mathbf{f}_{3}\right)=0.3 ; m\left(\mathbf{f}_{4}\right)=0.3 ; m\left(\mathbf{f}_{5}\right)=0.15 ; m\left(\mathbf{f}_{6}\right)=0.05$ (the same as the values in Figure 5). All the six cases are illustrated in Figure 6 and the truth of each case is shown in Table 1.

To sum up, Figure 7 depicts different frameworks corresponding to different cases (Cases 1-6).

Case 1. C-BOE + crisp set.

Case 2. C-BOE + fuzzy set.

Case 3. N-BOE + crisp set.

Case 4. N-BOE + fuzzy set.

Case 5. $\mathrm{M}-\mathrm{BOE}+$ crisp set.

Case 6. M-BOE + fuzzy set.

Firstly, Figure 7 displays that Cases 1-2 are traditionally expressed based on probability theory, while possibility theory is used to deal with Cases 3-4; secondly, Cases 1-4 are special cases of Cases 5-6, in that M-BOE is a mix of C$\mathrm{BOE}$ and $\mathrm{N}-\mathrm{BOE}$. Evidence theory is a generalized form to measure the truth of " $x$ is $A$ " which suits for Cases 1-6 all [27].

\section{An Integration of Evidence Theory and SSEP-CSHR}

3.1. The Two-Phase F-QFD. In practice, series of HOQs can be integrated into a multiphase QFD. Each phase is closely correlated in a multiphase QFD since the inputs of one phase needs to apply the outputs of the previous phase $[17,18]$. In the paper, we focused on QFD whose procedure has been thoroughly described by Mazur [10]. This approach consists of solving some successive HOQs. Among these HOQs, two HOQs named as Quality Deployment (QD) and Functional Deployment (FD) which is of the most essential are applied here to develop our MCDM model for SSEP-CSHR.

Quality Deployment (QD). It translates some Basic Requirements to certain quality attributes.

Functional Deployment (FD). It realized the quality attributes by specific alternatives.

At the beginning of the process, Demanded Qualities (DQs) have to be identified. The elements are gathered from surveys or experts' interviews. They are "WHATs" in QD phase:

$$
\mathbf{L}_{1}=\left\{R_{1}, \ldots, R_{i}, \ldots, R_{N_{1}}\right\}
$$

We determine the fuzzy importance ratings of DQs $\left(\mathbf{L}_{1}\right)$. The fuzzy importance ratings are usually obtained by subjective method discussed in Section 2.2. F-AHP, for example, Extent Analysis (EA) or Lambda-Max Method (LMM) can also be used to capture the fuzzy ratings $[6,25]$ :

$$
\widetilde{\mathbf{W}}^{\mathbf{L}_{1}}=\left(\widetilde{\mathbf{W}}_{1}^{\mathbf{L}_{1}}, \ldots, \widetilde{\mathbf{W}}_{i}^{\mathbf{L}_{1}}, \ldots, \widetilde{\mathbf{W}}_{N_{1}}^{\mathbf{L}_{1}}\right)
$$

DQs are translated into “Quality Attributes” (QAs). QAs $\left(\mathbf{L}_{2}\right)$ are assessment criteria that are related to the accomplishment of above DQs. Then QAs are "HOWs" in QD phase. And QFD team then generates the QAs as follows:

$$
\mathbf{L}_{2}=\left\{C_{1}, \ldots, C_{i}, \ldots, C_{N_{2}}\right\}
$$

Similarly, some Functional Techniques (FTs) $\left(\mathbf{L}_{3}\right)$, that is, our alternatives to realize above QAs, are further defined by panel of experts. Here QAs are "WHATs" for FD and FTs are "HOWs" for FD:

$$
\mathbf{L}_{3}=\left\{F_{1}, \ldots, F_{i}, \ldots, F_{N_{3}}\right\}
$$

The core elements of F-QFD are "relationships matrices" in $\mathrm{QD}$ and FD, respectively:

(1) The relationship matrix between DQs $\left(\mathbf{L}_{1}\right)$ and QAs $\left(\mathbf{L}_{2}\right)$ to express how a quality attribute to meet a requirement;

(2) The relationship matrix between QAs $\left(\mathbf{L}_{2}\right)$ and FTs $\left(\mathbf{L}_{3}\right)$ to express how an alternative to realize an attribute;

(3) The correlation matrix between DQs $\left(\mathbf{L}_{1}\right)$, QAs $\left(\mathbf{L}_{2}\right)$, and FTs $\left(\mathbf{L}_{3}\right)$ themselves.

To sum up, Table 2 defines all fuzzified inputs and outputs in the two-phase F-QFD.

All inputs and outputs are described by linguistic terms and defined as the fuzzy sets. A fuzzy set is fully and uniquely represented by its $\alpha$-cuts (see Table 3).

Chen and Ko $[17,18]$ gave a formulation to implement multiphase F-QFD. Wang et al. [20, 28] have demonstrated that the method is not proper and provided a set of nonlinear programming (NLP) models to do F-QFD.

Let $\widetilde{\mathbf{A}}$ and $\widetilde{\mathbf{B}}$ be two fuzzy numbers; operations on two fuzzy numbers are defined as

$$
\begin{aligned}
\widetilde{\mathbf{A}} * \widetilde{\mathbf{B}} & =\bigcup_{\alpha \in[0,1]} \alpha(\mathbf{A} * \mathbf{B})_{\alpha}=\bigcup_{\alpha \in[0,1]} \alpha\left(\mathbf{A}_{\alpha} * \mathbf{B}_{\alpha}\right) \\
& =\bigcup_{\alpha \in[0,1]} \alpha\left\{f f * \in g \mathbf{A}_{\alpha}, g \in \mathbf{B}_{\alpha}\right\} .
\end{aligned}
$$

Let $*$ denote any of the four basic arithmetic operations. $\mathbf{A}_{\alpha} * \mathbf{B}_{\alpha}$ follows arithmetic operations on closed intervals [27]. Then based on Chen et al. [17, 18], and Wang and Chin [20], an algorithm to solve the two-phase F-QFD is introduced. 


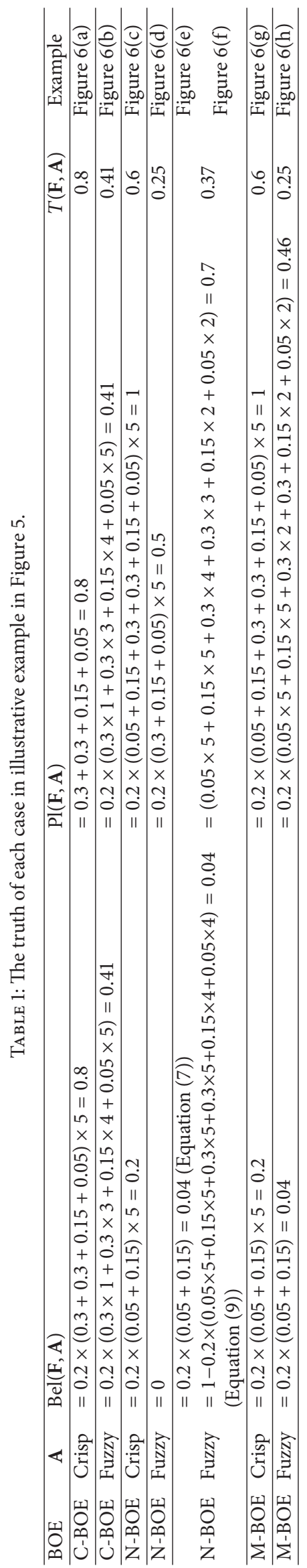



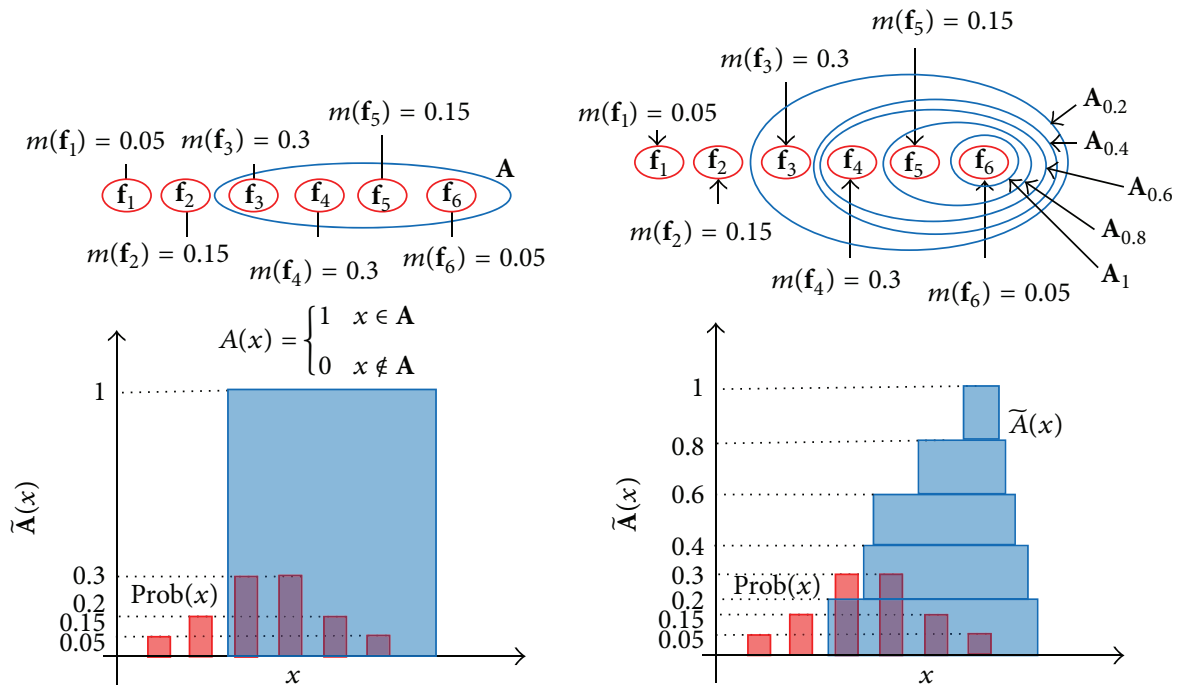

(a) C-BOE + crisp set

(b) C-BOE + fuzzy set
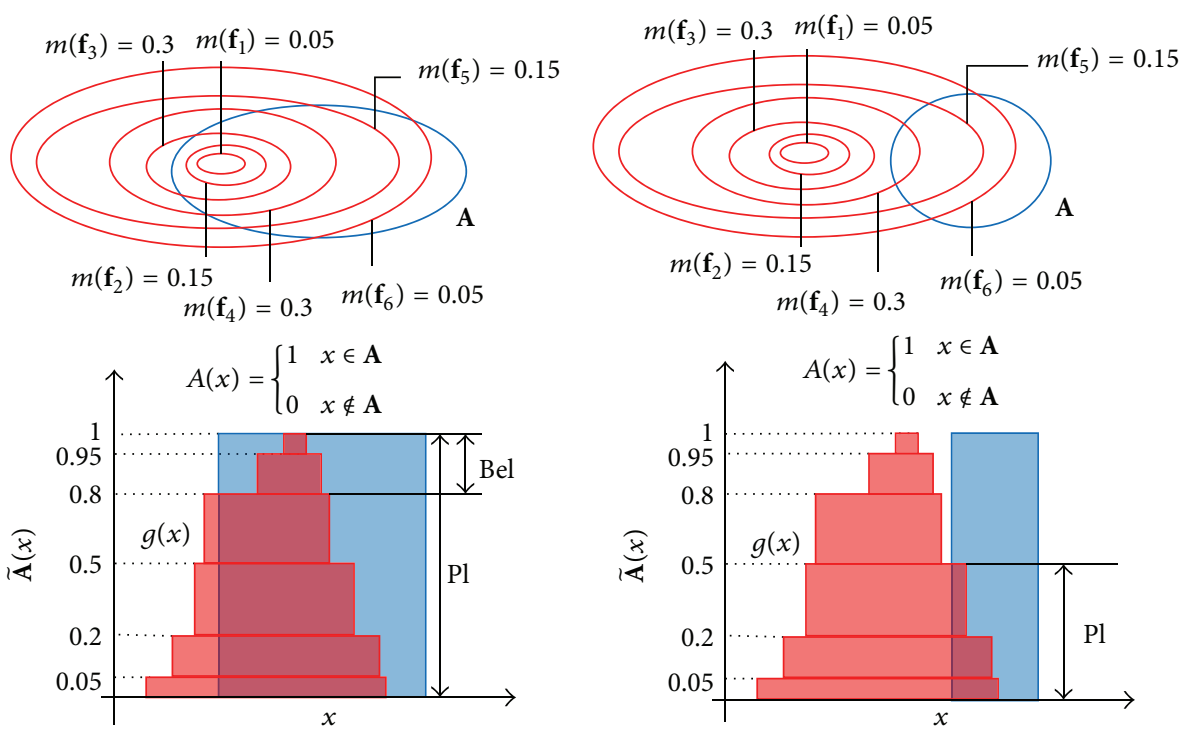

(c) $\mathrm{N}-\mathrm{BOE}+$ crisp set: the core of $g(x)$ is contained in $\mathbf{A}$

(d) N-BOE + crisp set: the core of $g(x)$ is not contained in $\mathbf{A}$
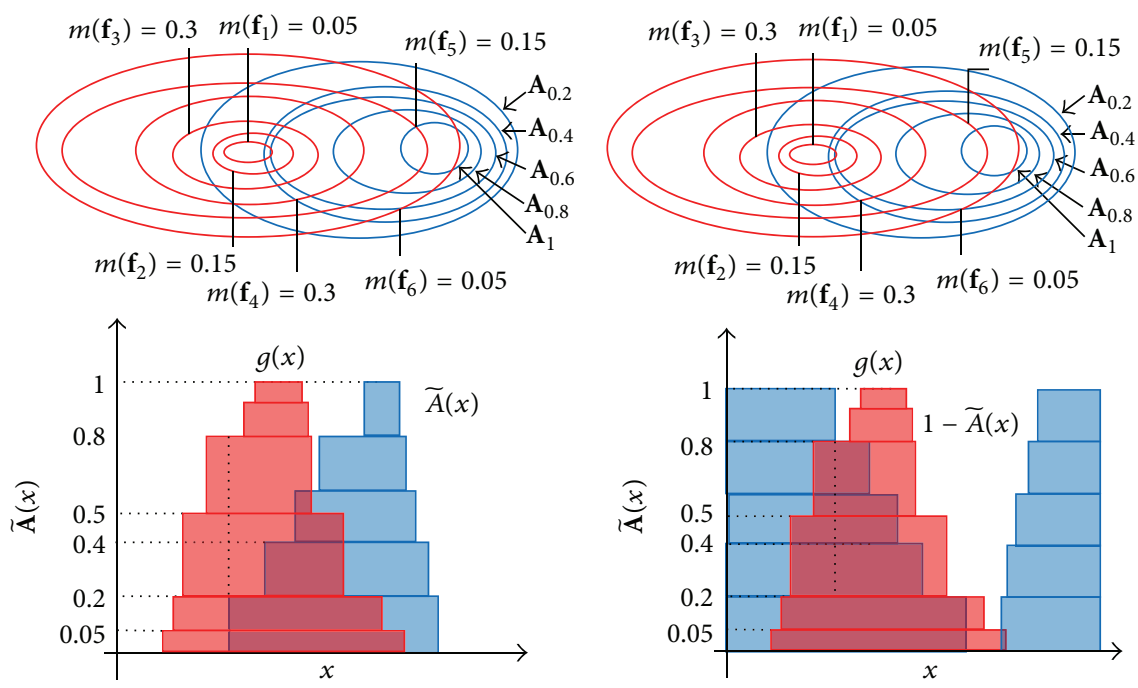

(e) N-BOE + fuzzy set: using (7) and (8)

(f) N-BOE + fuzzy set: using (9)

FIGURE 6: Continued. 

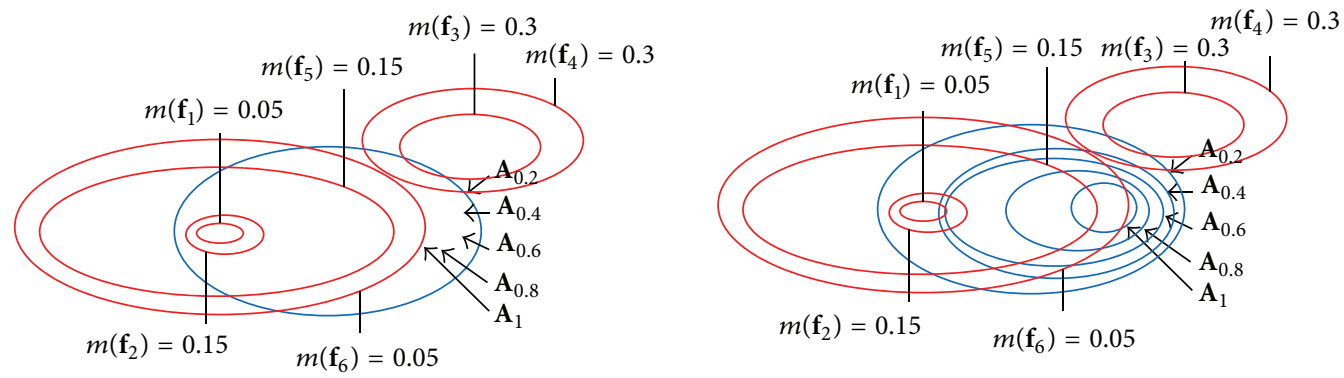

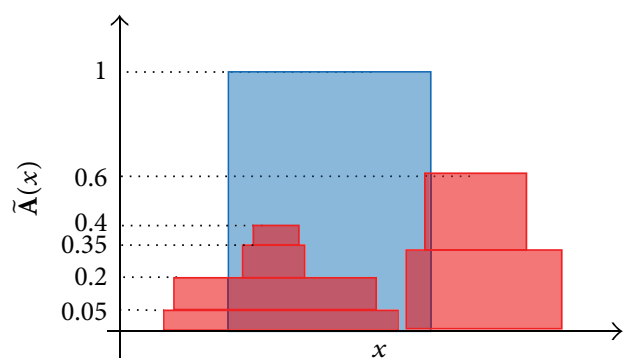

(g) $\mathrm{M}-\mathrm{BOE}+$ crisp set

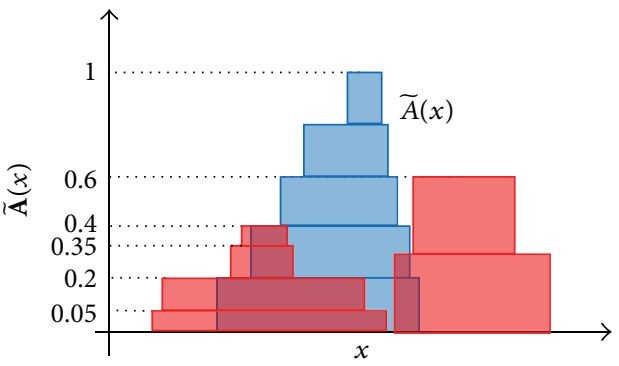

(h) M-BOE fuzzy set

FIgURE 6: Illustrative example of Belief and Plausibility measures.

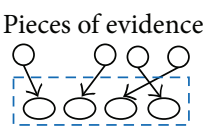

Pieces of evidence 0.98
1
1

Pieces of evidence

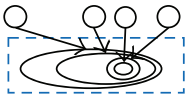

Pieces of evidence

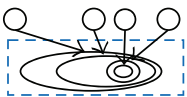

C-BOE

C-BOE

N-BOE

N-BOE
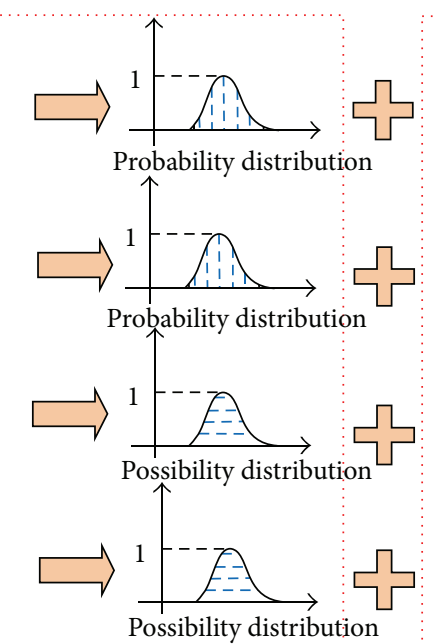

Possibility distribution

Pieces of evidence

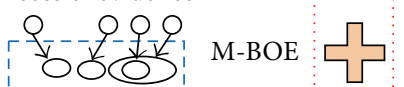

Pieces of evidence
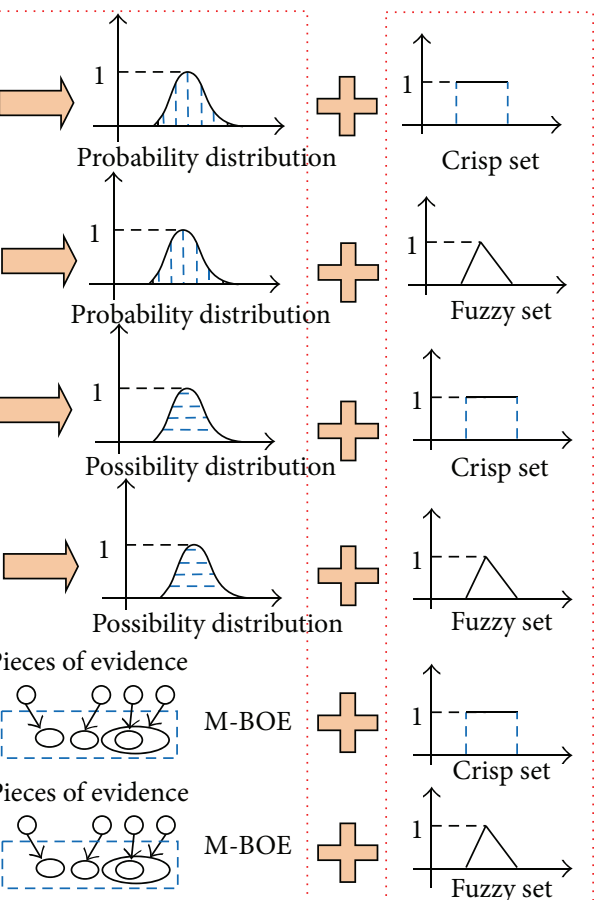

Case 6:

M-BOE + fuzzy set

Case 1:

C-BOE + crisp set

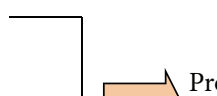

Case 2:

C-BOE + fuzzy set

Case 3:

N-BOE + crisp set

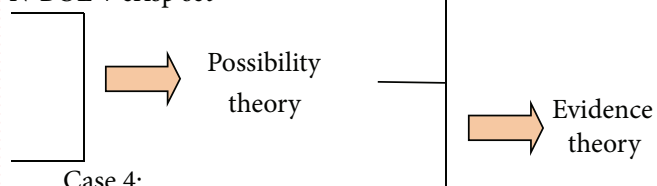

Case 5 : M-BOE + crisp set

FIGURE 7: Different theory corresponding to the combination of the type of BOE and the type of set $A$. 
TABLE 2: Inputs and outputs in HOQs of the two-phase F-QFD.

\begin{tabular}{ll}
\hline Inputs & Descriptions \\
\hline$\widetilde{\mathbf{W}}_{i}^{\mathbf{L}_{1}}$ & The fuzzy important ratings of $R_{i}$ where $i=1,2, \ldots, N_{1}$ \\
$\widetilde{\mathbf{B}}_{i j}^{\mathbf{L}_{2}}$ & The fuzzy relationship score between $R_{i}$ and $C_{j}$, where $i=1,2, \ldots, N_{1} ; j=1,2, \ldots, N_{2}$ \\
$\widetilde{\mathbf{B}}_{i j}^{\mathbf{L}_{3}}$ & The fuzzy relationship score between $C_{i}$ and $F_{j}$, where $i=1,2, \ldots, N_{2} ; j=1,2, \ldots, N_{3}$ \\
$\widetilde{\mathbf{A}}_{i j}^{\mathbf{L}_{1}}$ & The fuzzy correlation score between $R_{i}$ and $R_{j}$, where $i=1,2, \ldots, N_{1} ; j=1,2, \ldots, N_{1}$ \\
$\widetilde{\mathbf{A}}_{i j}^{\mathbf{L}_{2}}$ & The fuzzy correlation score between $C_{i}$ and $C_{j}$, where $i=1,2, \ldots, N_{2} ; j=1,2, \ldots, N_{2}$ \\
$\widetilde{\mathbf{A}}_{i j}^{\mathbf{L}_{3}}$ & The fuzzy correlation score between $F_{i}$ and $F_{j}$, where $i=1,2, \ldots, N_{3} ; j=1,2, \ldots, N_{3}$ \\
\hline Outputs & Descriptions \\
$\widetilde{\mathbf{W}}_{i}^{\mathbf{L}_{2}}$ & The fuzzy important ratings of $C_{i}$ where $i=1,2, \ldots, N_{2}$ \\
$\widetilde{\mathbf{W}}_{i}^{\mathbf{L}_{3}}$ & The fuzzy important ratings of $F_{i}$ where $i=1,2, \ldots, N_{3}$ \\
\hline
\end{tabular}

TABLE 3: The $\alpha$-cuts of the fuzzy sets in the two-phase F-QFD.

\begin{tabular}{ll}
\hline Notations & Descriptions \\
$\widetilde{\mathbf{W}}_{i \alpha}^{\mathbf{L}_{1}}=\left[\left(W_{i}^{\mathbf{L}_{1}}\right)_{\alpha}^{L},\left(W_{i}^{\mathbf{L}_{1}}\right)_{\alpha}^{U}\right]$ & The $\alpha$-cuts of fuzzy important ratings $\widetilde{\mathbf{W}}_{i}^{\mathbf{L}_{1}}$ \\
$\widetilde{\mathbf{B}}_{i j \alpha}^{\mathbf{L}_{2}}=\left[\left(B_{i j}^{\mathbf{L}_{2}}\right)_{\alpha}^{L},\left(B_{i j}^{\mathbf{L}_{2}}\right)_{\alpha}^{U}\right]$ & The $\alpha$-cuts of fuzzy relationship $\widetilde{\mathbf{B}}_{i j}^{\mathbf{L}_{2}}$ \\
$\widetilde{\mathbf{B}}_{i j \alpha}^{\mathbf{L}_{3}}=\left[\left(B_{i j}^{\mathbf{L}_{3}}\right)_{\alpha}^{L},\left(B_{i j}^{\mathbf{L}_{3}}\right)_{\alpha}^{U}\right]$ & The $\alpha$-cuts of fuzzy relationship $\widetilde{\mathbf{B}}_{i j}^{\mathbf{L}_{3}}$ \\
$\widetilde{\mathbf{A}}_{i j \alpha}^{\mathbf{L}_{1}}=\left[\left(A_{i j}^{\mathbf{L}_{1}}\right)_{\alpha}^{L},\left(A_{i j}^{\mathbf{L}_{1}}\right)_{\alpha}^{U}\right]$ & The $\alpha$-cuts of fuzzy correlations $\widetilde{\mathbf{A}}_{i j}^{\mathbf{L}_{1}}$ \\
$\widetilde{\mathbf{A}}_{i j \alpha}^{\mathbf{L}_{2}}=\left[\left(A_{i j}^{\mathbf{L}_{2}}\right)_{\alpha}^{L},\left(A_{i j}^{\mathbf{L}_{2}}\right)_{\alpha}^{U}\right]$ & The $\alpha$-cuts of fuzzy correlation $\widetilde{\mathbf{A}}_{i j}^{\mathbf{L}_{2}}$ \\
$\widetilde{\mathbf{A}}_{i j \alpha}^{\mathbf{L}_{3}}=\left[\left(A_{i j}^{\mathbf{L}_{3}}\right)_{\alpha}^{L},\left(A_{i j}^{\mathbf{L}_{3}}\right)_{\alpha}^{U}\right]$ & The $\alpha$-cuts of fuzzy correlation $\widetilde{\mathbf{A}}_{i j}^{\mathbf{L}_{3}}$ \\
$\widetilde{\mathbf{W}}_{i \alpha}^{\mathbf{L}_{2}}=\left[\left(W_{i}^{\mathbf{L}_{2}}\right)_{\alpha}^{L},\left(W_{i}^{\mathbf{L}_{2}}\right)_{\alpha}^{U}\right]$ & The $\alpha$-cuts of fuzzy important ratings $\widetilde{\mathbf{W}}_{i \alpha}^{\mathbf{L}_{2}}$ \\
$\widetilde{\mathbf{W}}_{i \alpha}^{\mathbf{L}_{3}}=\left[\left(W_{i}^{\mathbf{L}_{3}}\right)_{\alpha}^{L},\left(W_{i}^{\mathbf{L}_{3}}\right)_{\alpha}^{U}\right]$ & The $\alpha$-cuts of fuzzy important ratings $\widetilde{\mathbf{W}}_{i \alpha}^{\mathbf{L}_{3}}$ \\
\hline
\end{tabular}

Algorithm 3 (the algorithm to implement the two-phase F-QFD). Consider the following.

Step 1 . Set $\alpha$-cuts of the fuzzy set equal to $1 / K, \ldots$, and 1 respectively. $K$ is the number of $\alpha$-cut in $[0,1]$. Then the fuzzy importance ratings of FTs are obtained by following substeps.

Step 1.1. Let $k=1$.

$$
\begin{aligned}
\text { s.t } \quad\left(W_{i}^{\mathbf{L}_{k}}\right)_{\alpha}^{L} \leq \omega_{i}^{\mathbf{L}_{k}} \leq & \left(W_{i}^{\mathbf{L}_{k}}\right)_{\alpha}^{U} \\
& \forall i \in\left\{1,2, \ldots, N_{k}\right\}, \\
\left(A_{i j}^{\mathbf{L}_{k}}\right)_{\alpha}^{L} \leq a_{i j}^{\mathbf{L}_{k}} \leq & \left(A_{i j}^{\mathbf{L}_{k}}\right)_{\alpha}^{U} \\
& \forall i, j \in\left\{1,2, \ldots, N_{k}\right\} .
\end{aligned}
$$

Step 1.2. The fuzzy correlations between $\mathbf{L}_{k}$ can be normalized by formula:

$$
\widetilde{\omega}_{i}^{\mathbf{L}_{k}}=\frac{\sum_{n=1}^{N_{k}} \widetilde{W}_{n}^{\mathbf{L}_{k}} \otimes \widetilde{A}_{n i}^{\mathbf{L}_{k}}}{\sum_{n=1}^{N_{k}} \sum_{m=1}^{N_{k}} \widetilde{W}_{n}^{\mathbf{L}_{k}} \otimes \widetilde{A}_{n m}^{\mathbf{L}_{k}}} \quad \forall i \in\left\{1,2, \ldots, N_{k}\right\} .
$$

The formula can be achieved by the following Nonlinear Programming (NLP) models.

For all $i \in\left\{1,2, \ldots, N_{k}\right\}, \alpha \in[0,1]$, determine interval $\left[\left(\omega_{i}^{\mathbf{L}_{k}}\right)_{\alpha}^{L},\left(\omega_{i}^{\mathbf{L}_{k}}\right)_{\alpha}^{U}\right]$ by models:

(Model 1)

$$
\begin{gathered}
\left(\omega_{i}^{\mathbf{L}_{k}}\right)_{\alpha}^{L}=\min \quad \frac{\sum_{n=1}^{N_{k}} \omega_{n}^{\mathbf{L}_{k}} a_{n i}^{\mathbf{L}_{k}}}{\sum_{n=1}^{N_{k}} \sum_{m=1}^{N_{k}} \omega_{n}^{\mathbf{L}_{k}} a_{n m}^{\mathbf{L}_{k}}}, \\
\left(\omega_{i}^{\mathbf{L}_{k}}\right)_{\alpha}^{U}=\max
\end{gathered}
$$

Step 1.3. Furthermore, the fuzzy relationship between $\mathbf{L}_{k}$ and $\mathbf{L}_{k+1}$ can be defined by

$$
\widetilde{\mathbf{Y}}_{i}^{\mathbf{L}_{k+1}}=\frac{\sum_{\eta=1}^{N_{k+1}} \widetilde{\mathbf{B}}_{i \eta}^{\mathbf{L}_{k+1}} \otimes \widetilde{\mathbf{A}}_{\eta j}^{\mathbf{L}_{k+1}}}{\sum_{l=1}^{N_{k+1}} \sum_{\eta=1}^{N_{k+1}} \widetilde{\mathbf{B}}_{i \eta}^{\mathbf{L}_{k+1}} \otimes \widetilde{\mathbf{A}}_{\eta l}^{\mathbf{L}_{k+1}}}
$$

$$
\forall i \in\left\{1,2, \ldots, N_{k}\right\}
$$

Then we calculate the importance rating of $\mathbf{L}_{k+1}$ as the fuzzy weighted average of the normalized fuzzy relationships:

$$
\widetilde{\mathbf{W}}_{j}^{\mathbf{L}_{k+1}}=\frac{\sum_{i=1}^{N_{k}} \widetilde{\mathbf{W}}_{i}^{\mathbf{L}_{k}} \otimes \mathbf{Y}_{i j}^{\mathbf{L}_{k+1}}}{\sum_{i=1}^{N_{k}} \widetilde{\mathbf{W}}_{i}^{\mathbf{L}_{k}}} \quad \forall j \in\left\{1,2, \ldots, N_{k+1}\right\} .
$$

The above two formulas can be achieved by following NLP models.

For all $i \in\left\{1,2, \ldots, N_{k}\right\}, \alpha \in[0,1]$, we can determine interval: 
(Model 2)

$$
\begin{aligned}
& \left(W_{j}^{\mathbf{L}_{k+1}}\right)_{\alpha}^{L}=\min \frac{\sum_{\eta=1}^{N_{k}}\left(w_{i}^{\mathbf{L}_{k}} \sum_{\eta=1}^{N_{k+1}} b_{i \eta}^{\mathbf{L}_{k+1}} a_{\eta j}^{\mathbf{L}_{k+1}} / \sum_{l=1}^{N_{k+1}} \sum_{\eta=1}^{N_{k+1}} b_{i \eta}^{\mathbf{L}_{k+1}} a_{\eta l}^{\mathbf{L}_{k+1}}\right)}{\sum_{i=1}^{N_{k}} w_{i}^{\mathbf{L}_{k}}} \\
& \left(W_{j}^{\mathbf{L}_{k+1}}\right)_{\alpha}^{U}=\max \frac{\sum_{i=1}^{N_{k}}\left(w_{i}^{\mathbf{L}_{k}} \sum_{\eta=1}^{N_{k+1}} b_{i \eta}^{\mathbf{L}_{k+1}} a_{\eta j}^{\mathbf{L}_{k+1}} / \sum_{l=1}^{N_{k+1}} \sum_{\eta=1}^{N_{k+1}} b_{i \eta}^{\mathbf{L}_{k+1}} a_{\eta l}^{\mathbf{L}_{k+1}}\right)}{\sum_{i=1}^{N_{k}} w_{i}^{\mathbf{L}_{k}}} \\
& \text { s.t } \quad\left(A_{i j}^{\mathbf{L}_{k+1}}\right)_{\alpha}^{L} \leq a_{i j}^{\mathbf{L}_{k+1}} \leq\left(A_{i j}^{\mathbf{L}_{k+1}}\right)_{\alpha}^{U} \quad \forall i, j \in\left\{1,2, \ldots, N_{k+1}\right\} \\
& \left(\omega_{i}^{\mathbf{L}_{k}}\right)_{\alpha}^{L} \leq w_{i}^{\mathbf{L}_{k}} \leq\left(\omega_{i}^{\mathbf{L}_{k}}\right)_{\alpha}^{U} \quad \forall i \in\left\{1,2, \ldots, N_{k}\right\} \\
& \left(B_{i j}^{\mathbf{L}_{k+1}}\right)_{\alpha}^{L} \leq b_{i j}^{\mathbf{L}_{k+1}} \leq\left(B_{i j}^{\mathbf{L}_{k+1}}\right)_{\alpha}^{U} \quad \forall i \in\left\{1,2, \ldots, N_{k}\right\} \quad j \in\left\{1,2, \ldots, N_{k+1}\right\} .
\end{aligned}
$$

Step 1.3. By (1), the fuzzy importance ratings of $\mathbf{L}_{k+1}$ are obtained:

$$
\widetilde{\mathbf{W}}^{\mathbf{L}_{k+1}}=\left(\widetilde{\mathbf{W}}_{1}^{\mathbf{L}_{k+1}}, \ldots, \widetilde{\mathbf{W}}_{i}^{\mathbf{L}_{k+1}}, \ldots, \widetilde{\mathbf{W}}_{N_{k+1}}^{\mathbf{L}_{k+1}}\right) .
$$

Step 1.4. If $k+1=3$, go to Step 2; else $k=k+1$ return to Step 1.2 .

Step 2. Fuzzy importance rating can be ranked by different methods. In the paper, the fuzzy importance ratings are compared with each other by method developed in Wang [29]:

$$
\begin{array}{r}
\mathbf{W}_{i}^{\mathbf{L}_{k+1}}=\frac{1}{K} \sum_{\alpha \in[0,1]} \frac{\left(W_{i}^{\mathbf{L}_{k+1}}\right)_{\alpha}^{L}+\left(W_{i}^{\mathbf{L}_{k+1}}\right)_{\alpha}^{U}}{2} \\
\forall i \in\left\{1,2, \ldots, N_{k}\right\} .
\end{array}
$$

Step 3. END

Based on the method proposed in Wang and Chin [20], Models 1 and 2 can be linearized and solved optimally.

3.2. Service Station Evaluation Problem for CSHR. SSEPCSHR is based on a reinterpretation of above two-phase FQFD. In SSEP-CSHR, DQs in terms of Basic Requirements $\left(\mathbf{L}_{1}\right)$ are translated into QAs in terms of Assessment Criteria $\left(\mathbf{L}_{2}\right)$. And then service stations $\left(\mathbf{L}_{3}\right)$, that is, specific alternatives to realize above Assessment Criteria, are served as FTs. Figure 8 gives the corresponding relationship between the two-phase F-QFD and SSEP-CSHR.

Basic Requirements $\left(\mathbf{L}_{1}\right)$ in SSEP-CSHR are comprised of two aspects of requirements: Firstly, it contains some requirements used in FLE-F-QFD. These demands have been extensively described by the literatures about facility location and supply chain management $[11,19]$. Moreover, it involves some distinct requirements because of special CSHR background. For the sake of clarity, all those requirements are shown in Table 4.
Basic Requirements $\left(\mathbf{L}_{1}\right)$ are some requirements described by broad nature language. However, the set of Assessment Criteria $\left(\mathbf{L}_{2}\right)$ usually use more specific descriptions. Similarly Assessment Criteria $\left(\mathbf{L}_{2}\right)$ also consist of two parts: some criteria that are derived from relevant literatures [11, 19] and other ones that are gathered from experts' comments under certain background. All the criteria are presented in Table 5.

By solving the steps of the two-phase F-QFD (Algorithm 3), the importance ratings of alternative Service stations satisfying above requirements and criteria can be determined. Figure 9 provides the basic structure of two essential HOQs (QD and FD) used in SSEP-CSHR.

3.3. An Approximate Reasoning Procedure in SSEP-CSHR. Approximate reasoning is related to the appropriate processing of uncertain knowledge, given in a rule based manner. Deductive inference can be performed by means of classical logic modus ponens (MP). For using linguistic variables expressed fuzzy sets, a generalized MP has been proposed by Zadeh [30]. It enables an approximate deductive reasoning with the following general scheme:

$$
\begin{aligned}
& \text { Facts : } x \text { is } \widetilde{\mathbf{A}}^{\prime} \text { AND } y \text { is } \widetilde{\mathbf{B}}^{\prime} \\
& \text { Rule } 1 \text { : IF } x \text { is } \widetilde{\mathbf{A}}_{1} \text { AND } y \text { is } \widetilde{\mathbf{B}}_{1} \text { THEN } z \text { is } \widetilde{\mathbf{C}}_{1} \\
& \text { Rule } 2 \text { : IF } x \text { is } \widetilde{\mathbf{A}}_{2} \text { AND } y \text { is } \widetilde{\mathbf{B}}_{2} \text { THEN } z \text { is } \widetilde{\mathbf{C}}_{2}
\end{aligned}
$$$$
\text { Conclusions : } z \text { is } \widetilde{\mathbf{C}}^{\prime} \text {. }
$$

Let us proceed one step further and provide a generalization of the two-phase F-QFD based on the generalized MP. We find that the situation of "WHATs" in QD and "HOWs" in FD can all be described by propositions " $x$ is $\mathbf{A}$." The fundamental idea in our paper is to obtain the truth of the propositions about Basic Requirements by evidence theory to infer the truth of the propositions about service stations by implication "if $x$ is $\mathbf{A}$, then $y$ is $\mathbf{B}$." 
TABLE 4: List of $\mathbf{L}_{1}$ for SSEP-CSHR.

\begin{tabular}{|c|c|}
\hline Basic requirements & Description \\
\hline Proximity to trains $(\mathrm{PT})$ & Proximity to demands; number of trains that can be served; cost of serving trains \\
\hline Sound catering condition (SC) & Space and time condition for delivery \\
\hline Labor availability (LA) & $\begin{array}{l}\text { Availability of required professional and nonprofessional workforce; wage rates; quality of workforce; and } \\
\text { so forth }\end{array}$ \\
\hline Availability of foods (AF) & Closeness to food suppliers or distributional centers; freight costs of transportation \\
\hline Government incentives (GI) & Convenience provided by government or railway authorities \\
\hline Constructions costs (CC) & Construction costs of building cold stores at the station and also availability of space for future expansion \\
\hline
\end{tabular}

TABLE 5: List of $\mathbf{L}_{2}$ for SSEP-CSHR.

\begin{tabular}{|c|c|}
\hline Assessment criteria & Description \\
\hline $\begin{array}{l}\text { Quality and location of } \\
\text { neighboring suppliers (QL) }\end{array}$ & Proximity to suppliers; alternative suppliers; and speed and responsiveness of suppliers \\
\hline Accessibility to trains (AT) & $\begin{array}{l}\text { Number of train that can be served (number of trains departing from the city; number of trains } \\
\text { stopping at the city, the ratio of the above two) }\end{array}$ \\
\hline Available catering time (AC) & Average available catering time \\
\hline Physical catering conditions (PC) & The platform area \\
\hline $\begin{array}{l}\text { Human resource conditions } \\
\text { (HR) }\end{array}$ & Labor costs; attitudes toward works and labor turnover, economical growths, and declines effects \\
\hline Economics related factors $(\mathrm{EF})$ & Urban economic development, tax incentives, and business climate; interest rates \\
\hline Political issues (PI) & Availability and size of government; aids attitude of government to inward investment \\
\hline
\end{tabular}

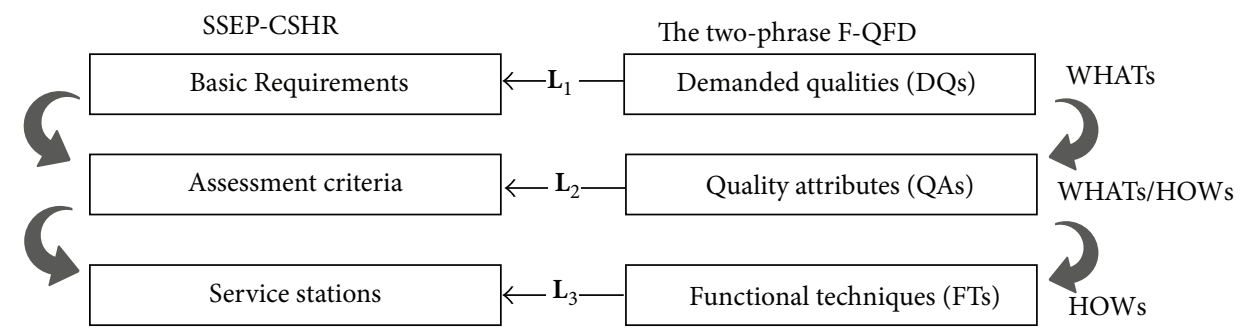

FIGURE 8: Corresponding relationship between the two-phase F-QFD and SSEP-CSHP.

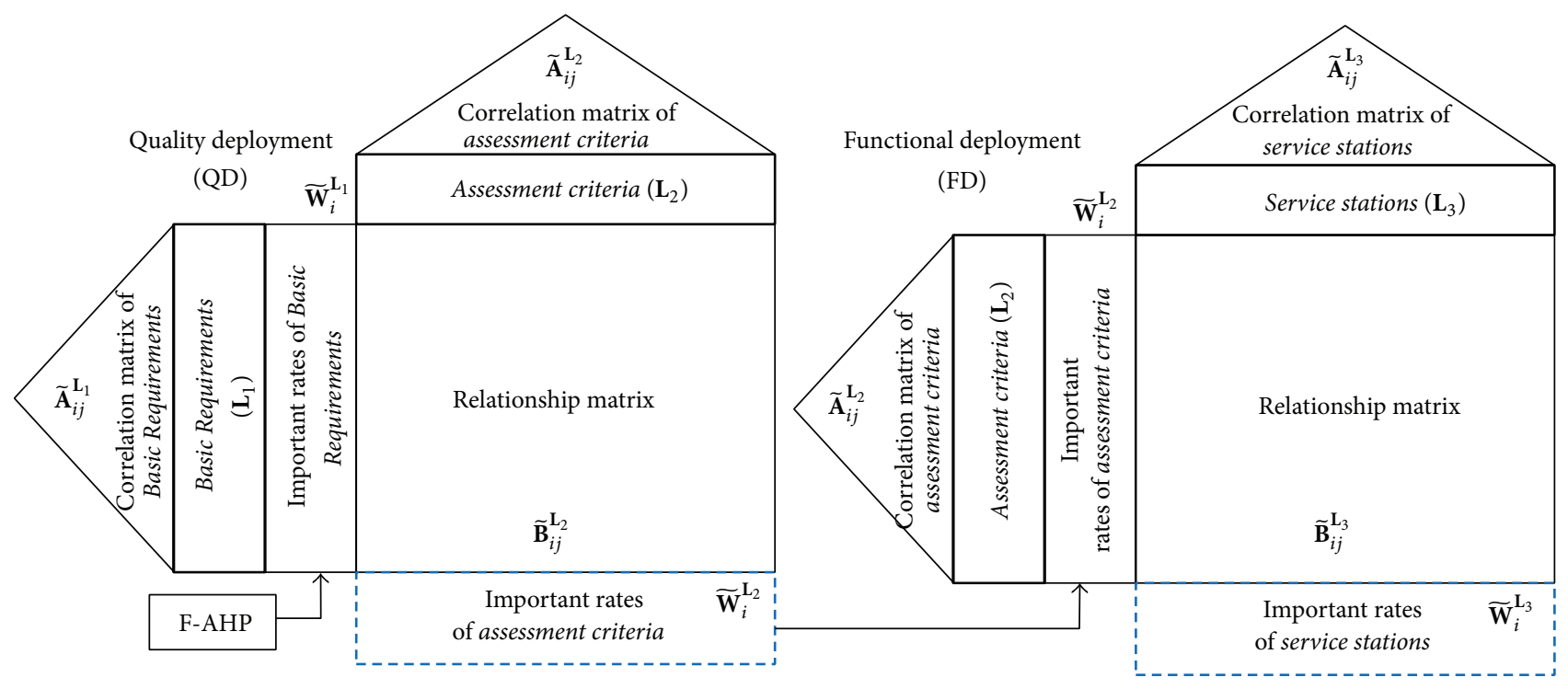

FIGURE 9: Basic structure of HOQs for SSEP-CSHR. 
The methods to generate the evidences $x$ and the linguistic terms of predicates in SSEP-CSHR are analyzed in Section 3.3.1. In Section 3.3.2, the expression of an if-then production rules and a general scheme of a multiconditional approximate reasoning in SSEP-CSHR are discussed.

3.3.1. Methods to Generate Evidence and Predicates. Figure 9 has provided the basic structure of two essential HOQs (QD and FD) used in SSEP-CSHR. Here we propose a method to generate the predicates of propositions incorporated in HOQs.

(1) Propositions about Basic Requirements $\mathbf{L}_{1}$. Column vector $\widetilde{\mathbf{W}}_{i}^{\mathbf{L}_{1}}$ in Figure 9 in QD shows the importance ratings of each $R_{i}$, that is, the following.

Proposition 4. $R_{i}$ is $\widetilde{\mathbf{W}}_{i}^{\mathbf{L}_{2}} \forall i \in\left\{1,2, \ldots, N_{1}\right\}$.

Proposition 4 can be truth-qualified by a canonical form " $x$ is $\mathbf{A}$ is $T$," where $T$ is a truth qualifier represented by crisp values. We should do the following analysis on truth-qualified proposition " $x$ is $\mathbf{A}$ is $T$ " [27] to capture the truth of " $x$ is $\mathbf{A}$."

Algorithm 5 (the Algorithm to capture the truth of " $x$ is A"). Consider the following

Step 1. The evidences about $R_{i}$ (that might be C-BOE, N-BOE, or $\mathrm{M}-\mathrm{BOE}$ ) are collected from many experts by questionnaires (the method to generate $\mathrm{BOE}$ has been discussed in Section 2.3).

For each $i \in\left\{1,2, \ldots, N_{1}\right\}$, a list of focal elements with basic probability distribution is then generated from the questionnaires (the list of focal elements is expressed by $\left.\left(\mathbf{F}_{i}^{\mathbf{L}_{1}}, m_{i}^{\mathbf{L}_{1}}\right)\right)$.

Step 2. The predicates in Proposition 4 are the same as the fuzzy importance ratings of $\mathbf{L}_{1}$ obtained by the two-phase FQFD (see Algorithm 3 Step 2).
Step 3. Then the truth of Proposition 4 is calculated by the following formulas:

$$
\begin{array}{r}
T\left(\mathbf{F}_{i}^{\mathbf{L}_{1}}, \widetilde{\mathbf{W}}_{i}^{\mathbf{L}_{1}}\right)=w_{1} \operatorname{Bel}\left(\mathbf{F}_{i}^{\mathbf{L}_{1}}, \widetilde{\mathbf{W}}_{i}^{\mathbf{L}_{1}}\right)+w_{2} \operatorname{Pl}\left(\mathbf{F}_{i}^{\mathbf{L}_{1}}, \widetilde{\mathbf{W}}_{i}^{\mathbf{L}_{1}}\right) \\
\forall i \in\left\{1,2, \ldots, N_{1}\right\},
\end{array}
$$

where $T\left(\mathbf{F}_{i}^{\mathbf{L}_{1}}, \widetilde{\mathbf{W}}_{i}^{\mathbf{L}_{1}}\right)$ is the truth qualifier of proposition " $R_{i}$ is

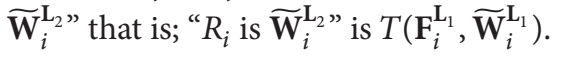

Step 4. End.

Table 6 shows the steps to analyze a truth-qualified proposition.

"Construction Cost is important' is true":

(i) The predicate "important" is given by a fuzzy set $\widetilde{\mathbf{W}}_{i}^{\mathbf{L}_{2}}$ (see Column 2).

(ii) The evidences about $x$ are generated from questionnaires (see Column 1).

(iii) The predicate "true" is expressed by a crisp number on $T\left(\mathbf{F}_{i}^{\mathbf{L}_{1}}, \widetilde{\mathbf{W}}_{i}^{\mathbf{L}_{1}}\right) \in[0,1]$ (see Column 3 ).

(2) Propositions about Service Stations $\left(\mathbf{L}_{3}\right)$. Row vector $\widetilde{\mathbf{W}}_{i}^{\mathbf{L}_{2}}$ in FD of Figure 9 shows the importance ratings of each $F_{i}$ (i.e., each station).

Proposition 6. $F_{i}$ is $\widetilde{\mathbf{W}}_{i}^{\mathbf{L}_{3}} \forall i \in\left\{1,2, \ldots, N_{3}\right\}$.

Here an approximate reasoning is demanded to infer the truth of Proposition 6 based on the truth of Proposition 4 (see Section 3.3.2).

3.3.2. Evidence-Based Inference Scheme in SSEP-CSHR. The evidence-based inference schema of multiconditional approximate reasoning in the two-phase F-QFD has the following form:

Facts: $R_{1}$ is $\mathbf{F}_{1}^{\mathbf{L}_{1}}$ AND $R_{2}$ is $\mathbf{F}_{2}^{\mathbf{L}_{1}}$ AND $\ldots R_{N_{1}}$ is $\mathbf{F}_{N_{1}}^{\mathbf{L}_{1}}$

Rule 1: IF $R_{1}$ is $\widetilde{\mathbf{W}}_{11}^{\mathrm{L}_{1}}$ AND $R_{2}$ is $\widetilde{\mathbf{W}}_{12}^{\mathrm{L}_{1}}$ AND $\ldots R_{N_{1}}$ is $\widetilde{\mathbf{W}}_{1 N_{1}}^{\mathrm{L}_{1}}$ THEN $F_{1}$ is $\widetilde{\mathbf{W}}_{11}^{\mathrm{L}_{3}}$ AND $F_{2}$ is $\widetilde{\mathbf{W}}_{12}^{\mathrm{L}_{3}}$ AND $\ldots F_{N_{3}}$ is $\widetilde{\mathbf{W}}_{1 N_{3}}^{\mathrm{L}_{3}}$

Rule 2: IF $R_{1}$ is $\widetilde{\mathbf{W}}_{21}^{\mathrm{L}_{1}}$ AND $R_{2}$ is $\widetilde{\mathbf{W}}_{21}^{\mathrm{L}_{1}}$ AND $\ldots R_{N_{1}}$ is $\widetilde{\mathbf{W}}_{2 N_{1}}^{\mathrm{L}_{1}}$ THEN $F_{1}$ is $\widetilde{\mathbf{W}}_{21}^{\mathrm{L}_{3}}$ AND $F_{2}$ is $\widetilde{\mathbf{W}}_{22}^{\mathrm{L}_{3}}$ AND $\ldots F_{N_{3}}$ is $\widetilde{\mathbf{W}}_{2 N_{3}}^{\mathrm{L}_{3}}$

$\frac{\text { Rule N: IF } R_{1} \text { is } \widetilde{\mathbf{W}}_{N 1}^{\mathrm{L}_{1}} \text { AND } R_{2} \text { is } \widetilde{\mathbf{W}}_{N 1}^{\mathrm{L}_{1}} \text { AND } \ldots R_{N_{1}} \text { is } \widetilde{\mathbf{W}}_{N N_{1}}^{\mathrm{L}_{1}} \text { THEN } F_{1} \text { is } \widetilde{\mathbf{W}}_{N 1}^{\mathrm{L}_{3}} \text { AND } F_{2} \text { is } \widetilde{\mathbf{W}}_{N 2}^{\mathrm{L}_{3}} \text { AND } \ldots F_{N_{3}} \text { is } \widetilde{\mathbf{W}}_{N N_{3}}^{\mathrm{L}_{3}}}{\text { Conclusions: } F_{1} \text { is } \widetilde{\mathbf{W}}_{1}^{\mathrm{L}_{3} \prime} \text { AND } F_{2} \text { is } \widetilde{\mathbf{W}}_{2}^{\mathrm{L}_{3} \prime} \text { AND } \ldots F_{N_{3}} \text { is } \widetilde{\mathbf{W}}_{N_{3}}^{\mathrm{L}_{3} \prime} .}$

Above inference schema developed to infer the truth of Proposition 6 consists of the following parts:

(1) The IF part of a rule consists of (i) some expressions (Proposition 4) linked by AND, and (ii) the fuzzy sets expressing the importance of the Basic Requirements in Rule $n$, for example:

$\widetilde{\mathbf{W}}_{n i}^{L_{1}}$ is the predicates describing the situation of $R_{i}$ by Rule $n$, 


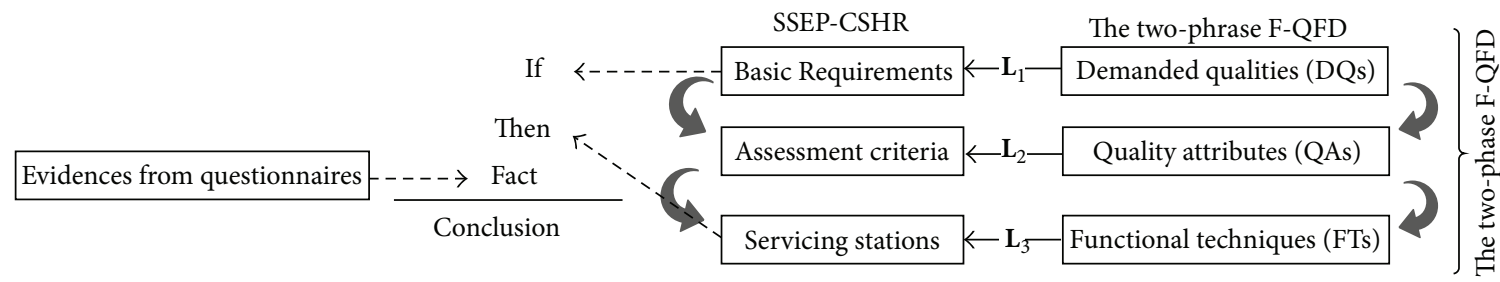

FIGURE 10: Corresponding relationship between SSEP-CSHR and proposed reasoning.

TABLE 6: An analysis on a truth-qualified proposition.

\begin{tabular}{|c|c|c|c|c|c|}
\hline & Column 1 & & Column 2 & & Column 3 \\
\hline Canonical form & $x$ & is & $\mathbf{A}$ & is & $\mathrm{T}$ \\
\hline Example & Construction cost & is & important & is & true \\
\hline Description & BOE expressing evidence about $x$ & & Fuzzy sets expressing predicates & & The truth of " $x$ is $\mathbf{A}$ " \\
\hline Math expression & $\left(\mathbf{F}_{i}^{\mathbf{L}_{1}}, m_{i}^{\mathbf{L}_{1}}\right)$ & & $\widetilde{\mathbf{W}}_{i}^{\mathbf{L}_{2}}$ & & $T\left(\mathbf{F}_{i}^{\mathbf{L}_{1}}, \widetilde{\mathbf{W}}_{i}^{\mathbf{L}_{1}}\right)$ \\
\hline Methods & $\begin{array}{l}\text { By organizing questionnaires from } \\
\text { experts }\end{array}$ & & $\begin{array}{l}\text { By estimation from QFD team (see } \\
\text { Algorithm 3) }\end{array}$ & & By (10) in evidence theory \\
\hline Analysis step & Step 1 in Algorithm 5 & & Step 2 in Algorithm 5 & & Step 3 in Algorithm 5 \\
\hline
\end{tabular}

(2) the THEN part of a rule consists of (i) some expressions linked by AND, and (ii) the fuzzy sets corresponding to the importance of the service stations in Rule $n$, for example,

$\widetilde{\mathbf{W}}_{n i}^{L_{3}}$ is the predicates describing the situation of $F_{i}$ by Rule $n$,

(3) given $N$ if-then rule, rules 1 though $N$, and Facts, Conclusions will be obtained,

$\mathbf{F}_{i}^{\mathbf{L}_{1}}$ is the evidences (BOE) describing the score range of $R_{i}$ obtained by questionnaires,

$\widetilde{\mathbf{W}}_{i}^{\mathbf{L}_{3}{ }^{\prime}}$ is a new predicate for $F_{i}$, which is modified by the truth of Proposition 6.

Figure 10 explicates the corresponding relationship between SSEP-CSHR and the above reasoning.

The most common way to determine conclusions in the above reasoning is referred to as a method of interpolation [27]. The interpolation method is actually a special case of the max-min compositional rule of inference that uses operations:

(1) intersection operation: minimization;

(2) implication operation: minimization;

(3) aggregations operation: maximization.

Based on the operations, we propose an algorithm as follows.
Algorithm 7 (the Algorithm to do approximate reasoning in SSEP-CSHR). Consider the following.

Step 1. Use Algorithm 3 to implement the two-phrase F-QFD in SSEP-CSHR.

Step 2. For each $i \in\left\{1,2, \ldots, N_{1}\right\}$, develop a BOE for $R_{i}$ by the method proposed in Section 2.3.

Step 3. For each $i \in\left\{1,2, \ldots, N_{1}\right\}, k \in\{1,2, \ldots, n\}$, by Algorithm 5, we calculate the truth of propositions:

$$
\begin{aligned}
T\left(\mathbf{F}_{i}^{\mathbf{L}_{1}}, \widetilde{\mathbf{W}}_{n i}^{\mathbf{L}_{1}}\right)= & w_{1} \operatorname{Bel}\left(\mathbf{F}_{i}^{\mathbf{L}_{1}}, \widetilde{\mathbf{W}}_{n i}^{\mathbf{L}_{1}}\right)+w_{2} \operatorname{Pl}\left(\mathbf{F}_{i}^{\mathbf{L}_{1}}, \widetilde{\mathbf{W}}_{n i}^{\mathbf{L}_{1}}\right) \\
& \forall i \in\left\{1,2, \ldots, N_{1}\right\}, n \in\{1,2, \ldots, N\} .
\end{aligned}
$$

The truth value here is a generalization of the concept of the degree of consistency in the interpolation method of multiconditional approximate reasoning [27].

Step 4. Calculate the degree of truth between the given facts and the antecedent of each if-then rule $n$ in terms of the fuzzy intersection operation:

$$
T_{n}=\min _{i \in\left\{1,2, \ldots, N_{1}\right\}}\left(T\left(\mathbf{F}_{i}^{\mathbf{L}_{1}}, \widetilde{\mathbf{W}}_{n i}^{\mathbf{L}_{1}}\right)\right) \quad \forall n \in\{1,2, \ldots, N\} .
$$

Step 5. By implication and aggregation operations above, we can calculate the Conclusions. That is,

$$
\begin{aligned}
\widetilde{\mathbf{W}}_{j}^{L_{3} \prime}(x)=\sup _{n \in\{1,2, \ldots, N\}} \min \left[T_{n}, \widetilde{\mathbf{W}}_{n j}^{L_{3}}(x)\right] & \\
& \forall j \in\left\{1,2, \ldots, N_{3}\right\} .
\end{aligned}
$$

for all $x \in \mathbf{U}$.

Step 6. End. 


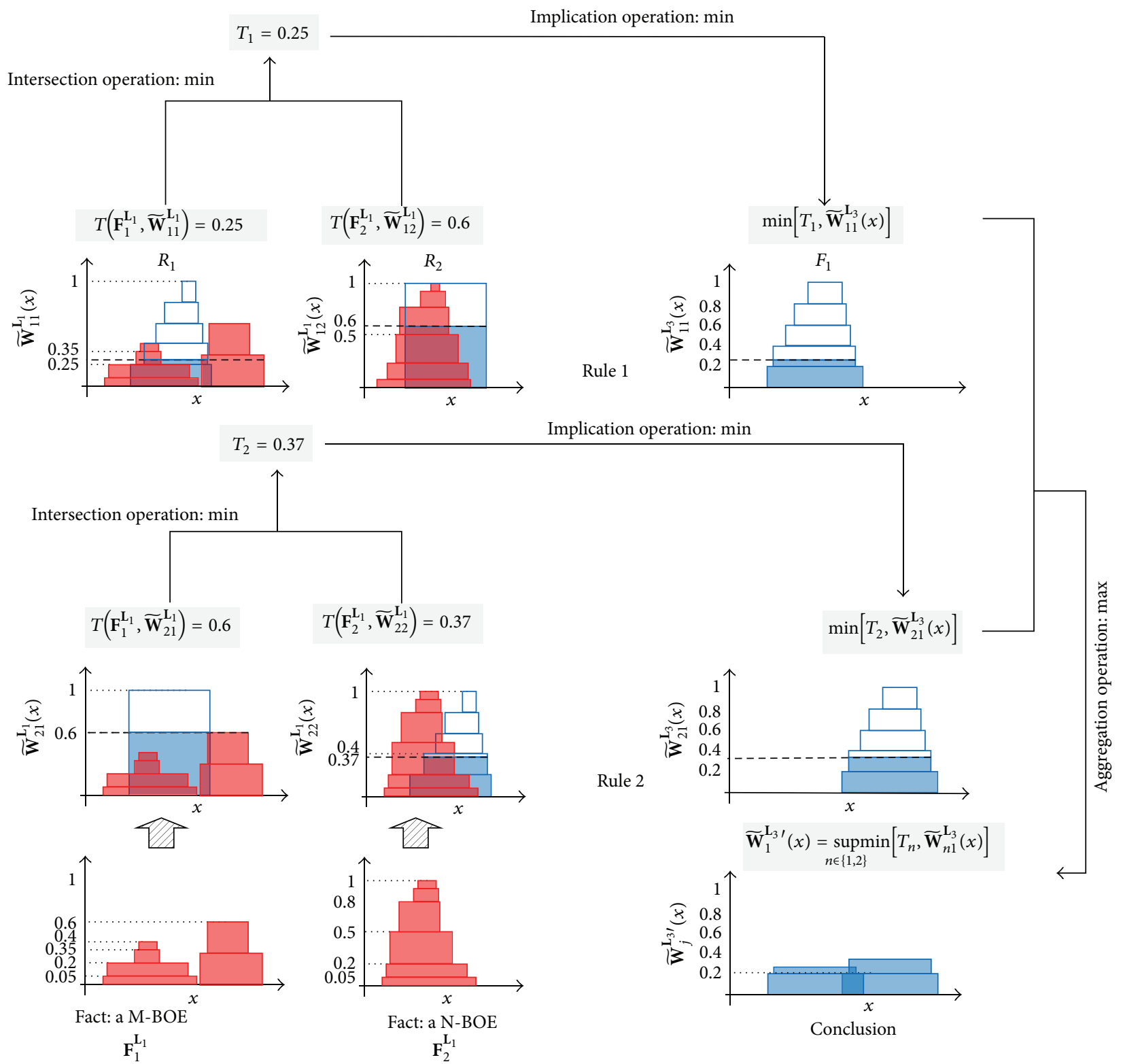

FIGURE 11: Illustration of approximate reasoning procedure in SSEP-CSHR.

An self-explanatory example of above approximate reasoning for two if-then rules with two Basic Requirements $\left(R_{1}, R_{2}\right)$ and a service station $\left(F_{1}\right)$ is given in Figure 11. In Figure 11 the fuzzy set, crisp set, and evidences about both $R_{1}$ and $R_{2}$ (a M-BOE and a N-BOE) have all been discussed in Section 2.4.

When BOE for each $i$ in $\left\{1,2, \ldots, N_{1}\right\}$ is a crisp number $a_{i}$,

$$
\begin{aligned}
T\left(a_{i}, \widetilde{\mathbf{W}}_{n i}^{\mathbf{L}_{1}}\right)=\widetilde{\mathbf{W}}_{n i}^{\mathbf{L}_{1}}\left(a_{i}\right) & \\
& \forall i \in\left\{1,2, \ldots, N_{1}\right\}, n \in\{1,2, \ldots, N\} .
\end{aligned}
$$

Then Algorithm 7 becomes a normal interpolation for a multiconditional approximate reasoning [27]. Figure 12 manifests this vestigial form.
The general steps of our integrated approach for SSEPCSHR are summarized in Figure 13.

It is noted that not only Proposition 4 but also any other propositions (of course containing the relation propositions abstract from Relation Matrices and Correlation Matrices in Figure 9) might serve as "cause" to affect the predicates of Proposition 6. However, in the above approximate reasoning we did not consider these "causes" for the following two reasons: Firstly, adding more "causes" did not influence the basic schema of above evidence-based reasoning. Secondly, the relationship between a "WHAT" and a "HOW" and the correlations of "WHATs" and "HOWs" are usually more "objective" (and thus truer) than the importance ratings of "WHATs." It means that the corresponding propositions of them are usually accompanied with higher truth value. If 


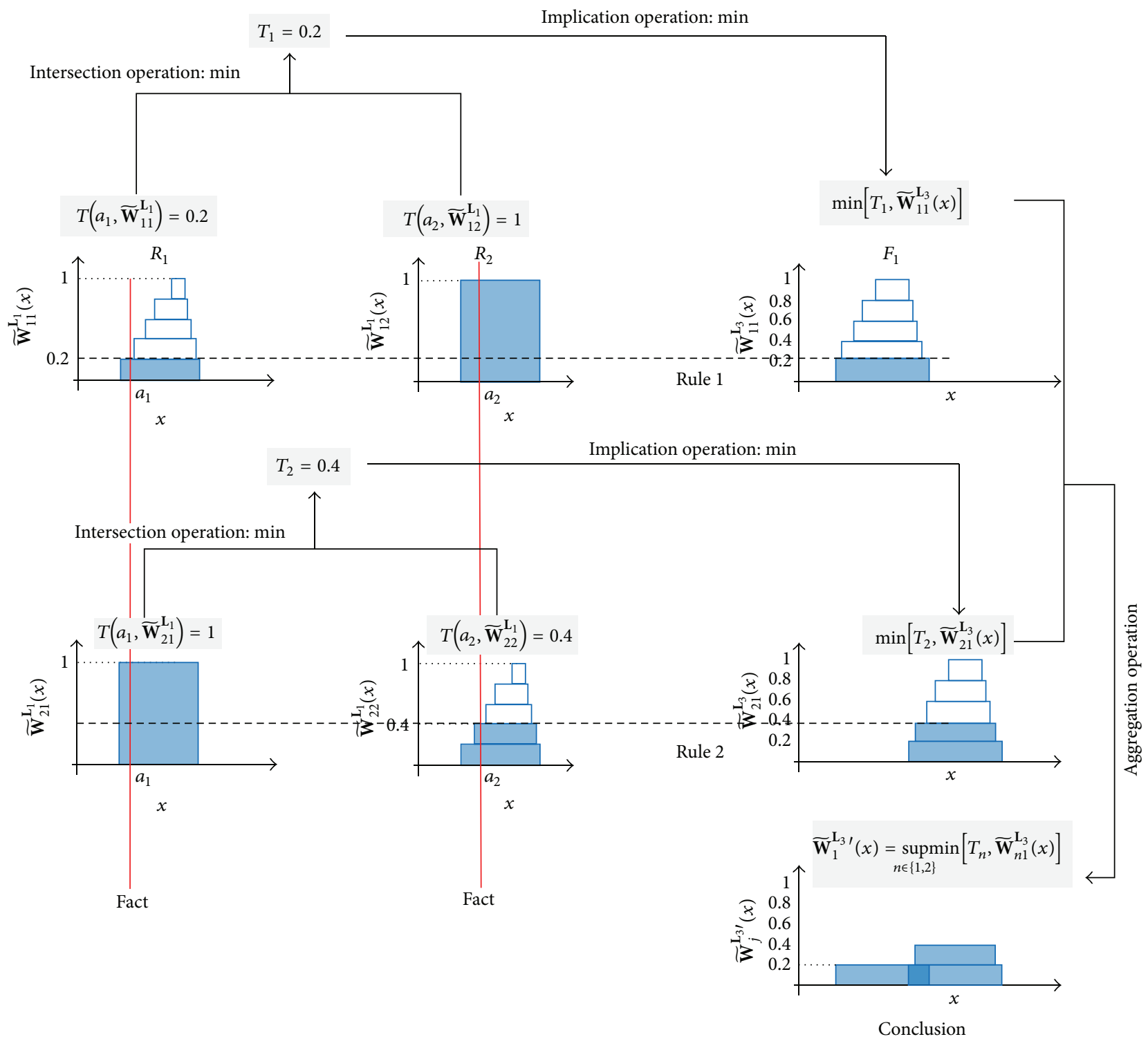

FIGURE 12: Illustration of normal interpolation for multiconditional approximate reasoning procedure.

standard fuzzy operations are used (as in Algorithm 7), these propositions only have little impact on the predicates of Conclusions.

\section{Model Implementation}

In this section, the integrated evidence theory and F-QFD for SSEP-CSHR will be applied to a practical case study, which refers to 85 alternative service stations on Chinese high-speed rail network. The set of service stations $\left(\mathbf{L}_{3}\right)$ is generated based on the real timetables of high-speed trains on April in 2014 in China (see Figure 14). The stations are selected from the rail stations with plenty of starting train lines and stations at which trains usually stop. Table 7 presents names of the stations and their indexes.
Tables 4 and 5 have presented $\mathbf{L}_{1}$ and $\mathbf{L}_{2}$ by details. Further QFD team expresses the opinions about the relationship between $\mathbf{L}_{1}$ and $\mathbf{L}_{2}$ in Table 8 (i.e., the relationship matrix in QD). The relationship between $\mathbf{L}_{2}$ and $\mathbf{L}_{3}$ is expressed in Appendix ((i.e., the relationship matrix in FD)). Correlation degrees between $\mathbf{L}_{1}, \mathbf{L}_{2}$, and $\mathbf{L}_{3}$ themselves are not considered in this study for simplification. In all the following matrices, $a / b / c$ means a triangle fuzzy number on an $x$ - $\alpha$ graph; say $\alpha=1$ with $x=b$, and say $\alpha=0$ with $x \in[a, c]$.

Table 9 is a pairwise comparison matrix of F-AHP used here to give the importance ratings of Basic Requirements. Three F-AHPs are used to determine the importance rating of Basic Requirements $\mathbf{L}_{1}$ including Extent Analysis (EA) [6], Modified Extent Analysis (MEA) [24], and Lambda-Max Method (LMM) [25]. Different QFD teams might generate 


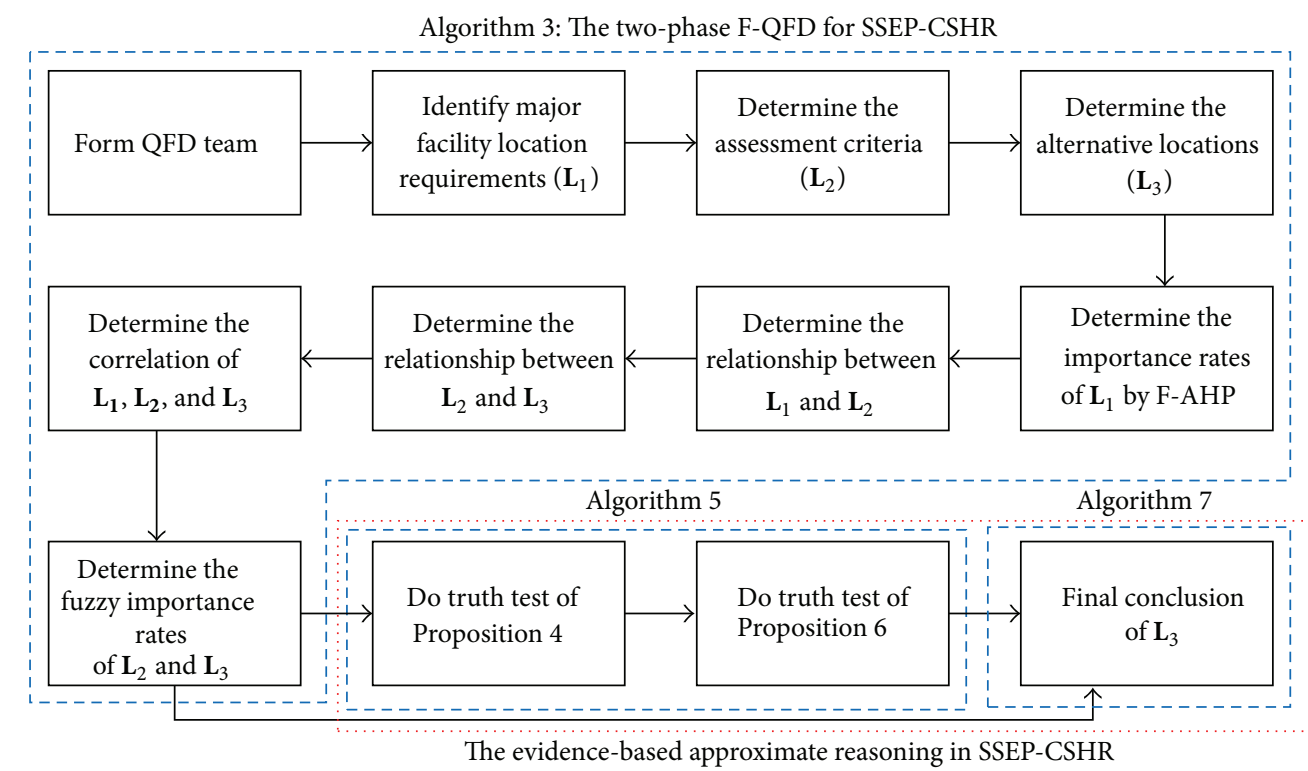

FIGURE 13: General steps of proposed approach.

different comparison matrix of F-AHP and thus generate different production rules in approximate reasoning. In this case, only one QFD team has made our decision.

Table 10 shows the fuzzy importance ratings of requirements in $\mathrm{L}_{1}$ obtained by EA, MEA, and LMM. By using (25) to defuzzify the fuzzy importance ratings, all three methods obtain the crisp results that signify " $\mathrm{SC}>\mathrm{PT}>\mathrm{CC}>\mathrm{LA}>$ GI > AF". We also find that the fuzzy importance ratings obtained by LMM are "narrower" than the ratings from MEA and EA. Klir and Yuan [27] characterize this nature as nonspecificity (Non). Then we get $\operatorname{Non}_{\mathrm{EA}}>\mathrm{Non}_{\mathrm{MEA}}>$ Non $_{\text {LMM }}$.

$\mathrm{N}$-BOEs are generated randomly with 20,000 samples corresponding to each $R_{i}$ in $\mathrm{L}_{1}$. Based on the N-BOE and the fuzzy importance ratings in Table 10, we can measure the truth between the evidences about Basic Requirements and the predicates of Proposition 4 (which are obtained by fuzzy importance ratings of $\mathbf{L}_{1}$ ). In our experiment, N-BOEs are used instead of M-BOE for the purpose of easy presentation. Table 11 shows that fuzzy importance ratings calculated by LMM have lower truth than the results by EA and MEA, that is Truth $_{\mathrm{EA}}>$ Truth $_{\mathrm{MEA}}>$ Truth $_{\mathrm{LMM}}$. It is just the opposite of the state of nonspecificity. It follows that vagueness usually leads propositions more difficult to be falsified.

Figure 15 explicitly illustrates the relationship between the fuzzy important ratings (or the predicates of Proposition 4) obtained by EA, MEA, and LMM and the N-BOE of PT. Apparently, the predicates obtained by LMM are too strict to be proved by our evidences (Bel measure is 0 and $\mathrm{Pl}$ measure is 1). The fuzzy important rate of PT from EA although hardly provides any precision information, in this case, but

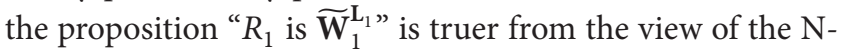
BOE. The values of "Min Truth" in Table 11 are important for that they determine the truth of our results of SSEP-CSHR when we only have one Rule in evidence-based inference schema.

Figure 16 displays the trade-off between nonspecificity and truth of fuzzy important ratings in F-QFD by a fitting curve based on all the fuzzy important ratings obtained by the above three methods. It actually shows an inclination that truer propositions are usually accompanied with more Fuzziness. It is noted that Klir and Yuan [27] treat fuzziness and nonspecificity as different conceptions; however, in many researches, nonspecificity is actually used the same as Fuzziness, such as Csutora and Buckley [25], and in fact, in general case nonspecificity is normally directly proportional to fuzziness.

It leads to some interesting conclusions in the field of "uncertainty management." In many existing literatures, minimizing Fuzziness usually serves as a basic principle in MCDM problems. For example, Csutora and Buckley [25] designed LMM in basis of the principle of minimizing Fuzziness. Wang et al. [20, 24] criticized the normalization methods in Chang's [6] EA and Chen et al's (2011) fuzzy linear programming because they did not generate the narrowest interval and fuzzy weights.

Our experiments partly justify a diversity of "uncertainty management," just as Klir and Yuan discussed in their book [27]. In F-QFD, when we use precise predicates to describe the situation of $\mathbf{L}_{1}$, it is possible (not necessary) to result in less truth Conclusions in reasoning and thus decrease the credibility of our evaluation.

Using the results of LMM as inputs, then we execute our two-phase F-QFD methods. Let $K$ be the number of $\alpha$-cut in $[0,1]$. When solving the NLPs, that is, (15)-(19), we define $K=10$. Above algorithms are all coded in MATLAB and executed by using a desktop with CPU Intel-Core i7-4700MQ and $8 \mathrm{G}$ RAM. Finally using about $15 \mathrm{~min}$ we get the fuzzy important ratings of 85 stations. 


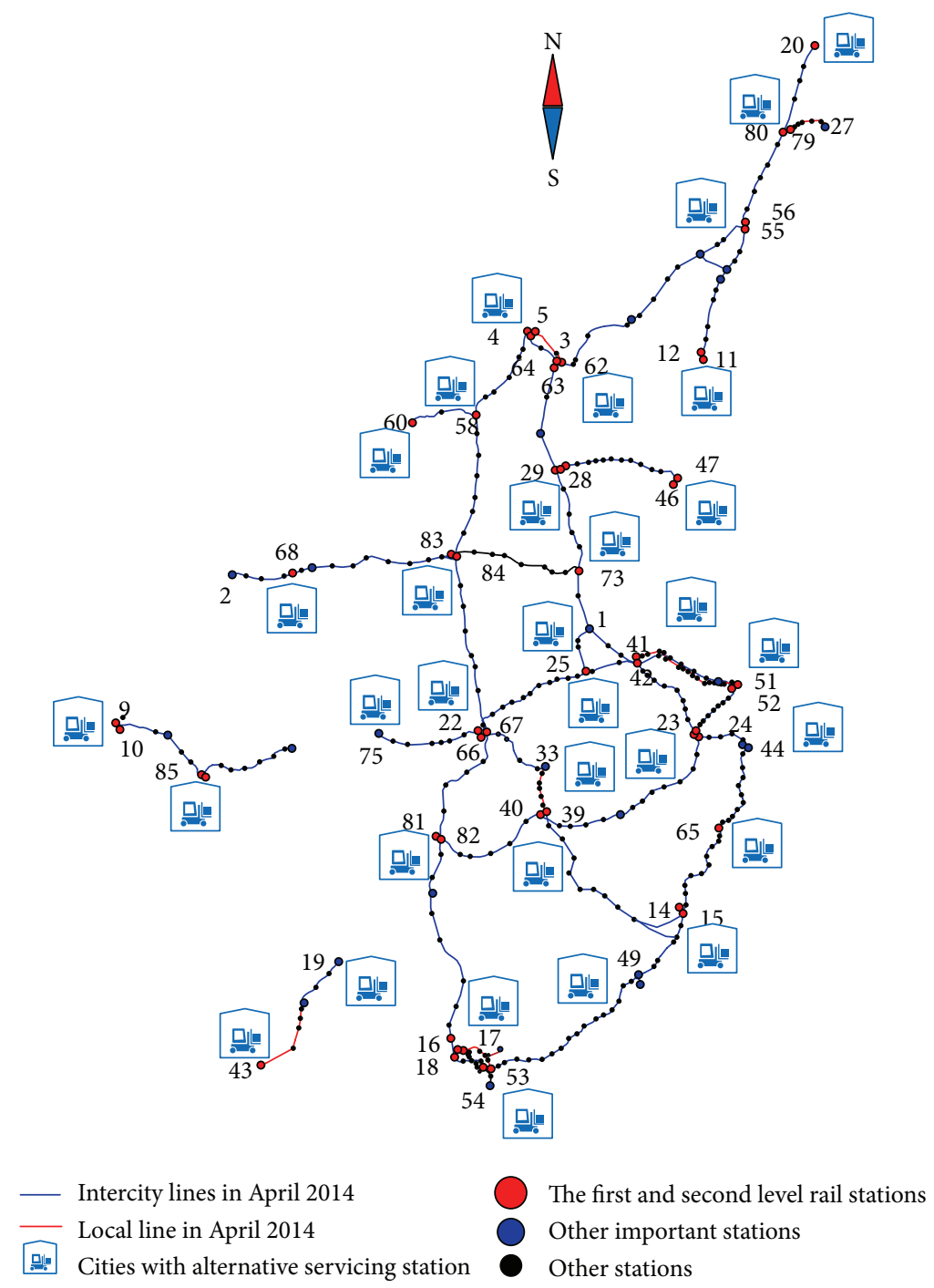

FIGURE 14: Alternative service stations considered in our case study.

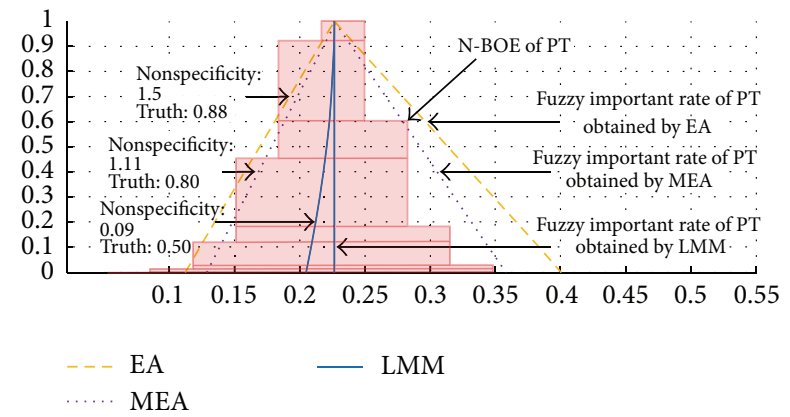

Figure 15: Fuzzy important rate of $\mathrm{PT}$ in $\mathbf{L}_{1}$ by three different methods.

4.1. Small Scale Example. We use the data in Appendix (ID: $1,5,9,18,22,42,52,68)$ to construct a $7 * 8$ relationship matrix between QAs and these eight service stations. Then by Algorithm 3, based on the outputs of phase 1, we determine the fuzzy important rating of stations. Due to large amount of outputs, only eight stations' important ratings at certain $\alpha$ cuts $(\alpha=0$ and $\alpha=1)$ are displayed in Table 12. The fuzzy sets which are used to express the fuzzy important ratings are shown in Figure 17(a). Figure 17(b) shows the Conclusions obtained by proposed approximate reasoning.

Figure 18 gives all fuzzy important ratings of above eight service stations (with lower and upper bounds when $\alpha=0$, as well as the core of the fuzzy sets) based on all the above three types of F-AHPs (i.e., EA, MEA, and LMM). We find that the Conclusions obtained based on LMM provide the most precision information, but with only "truth value $=0.25$." The Conclusions obtained from EA provide the most vague outputs, however, with "truth value $=0.48$."

4.2. Large Scale Example. Figure 19 utilizes a boxplot to display all the fuzzy important ratings of above 85 stations $\left(\mathbf{L}_{3}\right)$ $(a / b / c$, the minimum of a box is $a$ in fuzzy triangle number $a / b / c$; the median of a box is $b$ in fuzzy triangle number 


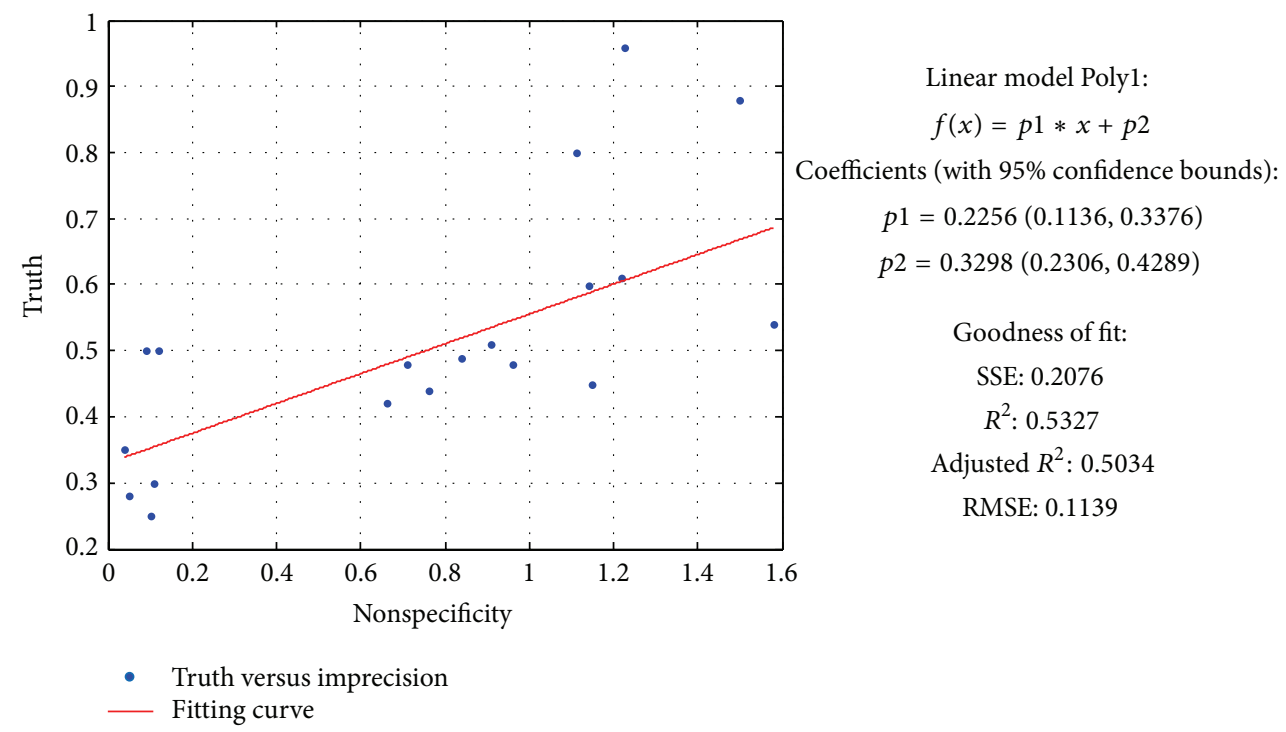

FIGURE 16: Trade-off between imprecision and truth of fuzzy important ratings.
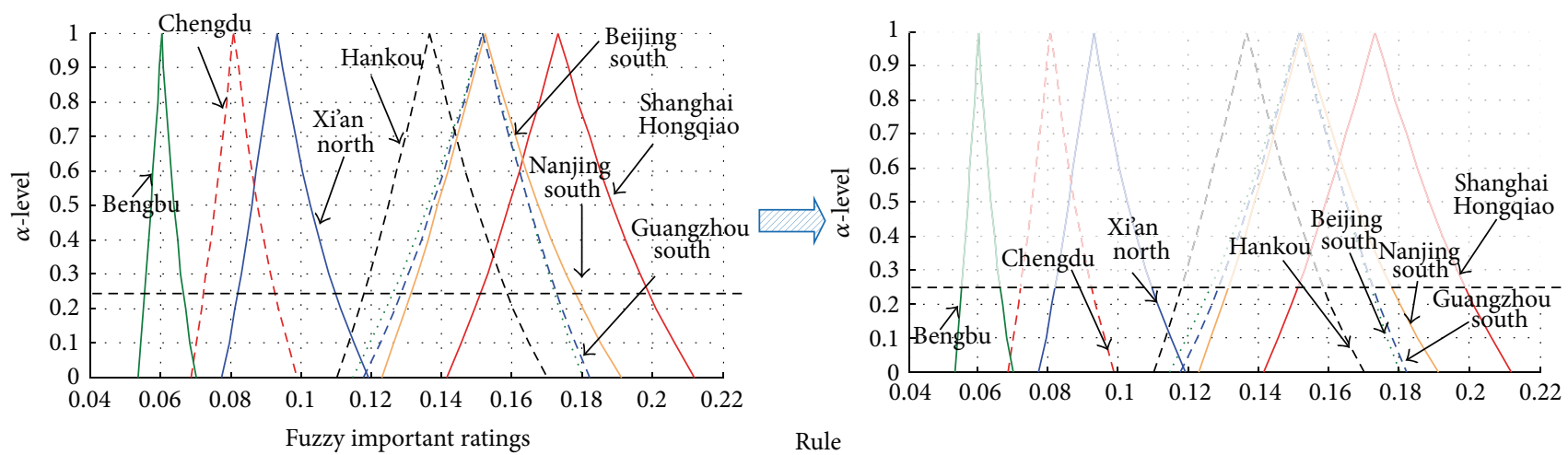

FIGURE 17: Fuzzy important ratings of the eight service stations and the conclusions after reasoning.

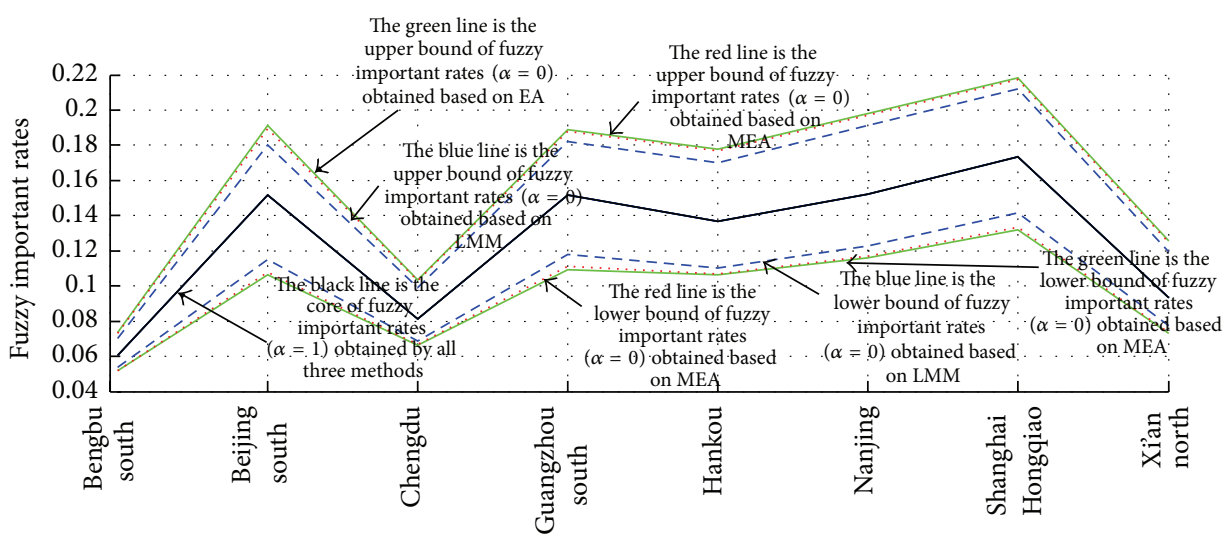

FIGURE 18: Fuzzy important ratings of service stations based on different F-AHPs. 
TABLE 7: Alterative service rail stations and their IDs in example network.

\begin{tabular}{|c|c|}
\hline ID & Station \\
\hline 1 & Bengbu south \\
\hline 2 & Baoji south \\
\hline 3 & Beidaihe \\
\hline 4 & Beijing \\
\hline 5 & Beijing south \\
\hline 6 & Beijing west \\
\hline 7 & Cangnan \\
\hline 8 & Changzhou \\
\hline 9 & Chengdu \\
\hline 10 & Chengdu east \\
\hline 11 & Dalian \\
\hline 12 & Dalian north \\
\hline 13 & Fuding \\
\hline 14 & Fuzhou \\
\hline 15 & Fuzhou south \\
\hline 16 & Guangzhou \\
\hline 17 & Guangzhou east \\
\hline 18 & Guangzhou south \\
\hline 19 & Guilin \\
\hline 20 & Harbin west \\
\hline 21 & Handan east \\
\hline 22 & Hankou \\
\hline 23 & Hangzhou \\
\hline 24 & Hangzhou east \\
\hline 25 & Hefe \\
\hline 26 & Huainan east \\
\hline 27 & Jilin \\
\hline 28 & Jinan \\
\hline 29 & Jinan west \\
\hline 30 & Jiangshan \\
\hline 31 & Jinhua west \\
\hline 32 & Jingzhou \\
\hline 33 & Jiujiang \\
\hline 34 & Lichuan \\
\hline 35 & Lingbao west \\
\hline 36 & Liu'an \\
\hline 37 & Longyan \\
\hline 38 & Luoyang longmen \\
\hline 39 & Nanchang \\
\hline 40 & Nanchang west \\
\hline 41 & Nanjing \\
\hline 42 & Nanjing south \\
\hline 43 & Nanning \\
\hline 44 & Ningbo \\
\hline 45 & Qinhuangdao \\
\hline 46 & Qingdao \\
\hline 47 & Qingdao north \\
\hline 48 & Sanming north \\
\hline 49 & Xiamen north \\
\hline
\end{tabular}

TABle 7: Continued.

\begin{tabular}{|c|c|}
\hline ID & Station \\
\hline 50 & Shangqiu \\
\hline 51 & Shanghai \\
\hline 52 & Shanghai Hongqiao \\
\hline 53 & Shenzhen \\
\hline 54 & Shenzhen north \\
\hline 55 & Shenyang \\
\hline 56 & Shenyang north \\
\hline 57 & Shiyan \\
\hline 58 & Shijiazhuang \\
\hline 59 & Suzhou \\
\hline 60 & Taiyuan \\
\hline 61 & Taining \\
\hline 62 & Tianjin \\
\hline 63 & Tianjin south \\
\hline 64 & Tianjin west \\
\hline 65 & Wenzhou south \\
\hline 66 & Wuchang \\
\hline 67 & Wuhan \\
\hline 68 & Xian north \\
\hline 69 & Xiangyang \\
\hline 70 & Xinxiang east \\
\hline 71 & Xinyang east \\
\hline 72 & Xuzhou \\
\hline 73 & Xuzhou east \\
\hline 74 & Yan'an \\
\hline 75 & Yichang east \\
\hline 76 & Yiwu \\
\hline 77 & YueYangDong \\
\hline 78 & Zhangzhou \\
\hline 79 & Changchun \\
\hline 80 & Changchun west \\
\hline 81 & Changsha \\
\hline 82 & Changsha south \\
\hline 83 & Zhengzhou \\
\hline 84 & Zhengzhou east \\
\hline 85 & Chongqing north \\
\hline
\end{tabular}

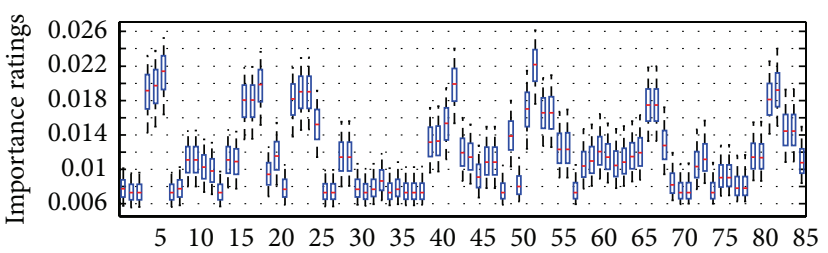

The indexes of service stations

FIGURE 19: Boxplots to express the fuzzy important ratings for service stations. 
TABLE 8: Relation matrices of QD (between $\mathbf{L}_{1}$ and $\mathbf{L}_{2}$ ).

\begin{tabular}{|c|c|c|c|c|c|c|c|}
\hline & QL & AT & $\mathrm{AC}$ & $\mathrm{PC}$ & HR & $\mathrm{EF}$ & PI \\
\hline PT & $0.08 / 0.09 / 0.1$ & $0.29 / 0.29 / 0.29$ & $0.1 / 0.12 / 0.14$ & $0.11 / 0.12 / 0.15$ & $0.08 / 0.09 / 0.1$ & $0.11 / 0.12 / 0.15$ & $0.16 / 0.17 / 0.19$ \\
\hline SC & $0.07 / 0.08 / 0.08$ & $0.07 / 0.08 / 0.08$ & $0.24 / 0.25 / 0.25$ & $0.24 / 0.25 / 0.26$ & $0.07 / 0.08 / 0.09$ & $0.13 / 0.14 / 0.15$ & $0.13 / 0.14 / 0.15$ \\
\hline LA & $0.08 / 0.09 / 0.11$ & $0.11 / 0.14 / 0.16$ & $0.08 / 0.09 / 0.12$ & $0.09 / 0.09 / 0.12$ & $0.31 / 0.31 / 0.31$ & $0.11 / 0.14 / 0.16$ & $0.12 / 0.14 / 0.17$ \\
\hline $\mathrm{AF}$ & $0.3 / 0.3 / 0.3$ & 0.08/0.09/0.11 & $0.1 / 0.13 / 0.15$ & $0.11 / 0.13 / 0.16$ & $0.08 / 0.09 / 0.11$ & $0.11 / 0.13 / 0.16$ & $0.11 / 0.13 / 0.17$ \\
\hline GI & $0.13 / 0.15 / 0.17$ & $0.13 / 0.15 / 0.18$ & $0.08 / 0.09 / 0.11$ & $0.08 / 0.09 / 0.12$ & $0.06 / 0.07 / 0.08$ & $0.26 / 0.26 / 0.26$ & $0.18 / 0.2 / 0.23$ \\
\hline $\mathrm{CC}$ & $0.15 / 0.17 / 0.18$ & $0.15 / 0.17 / 0.19$ & $0.1 / 0.12 / 0.13$ & $0.1 / 0.12 / 0.14$ & $0.08 / 0.08 / 0.09$ & $0.22 / 0.23 / 0.23$ & $0.11 / 0.12 / 0.14$ \\
\hline
\end{tabular}

TABLE 9: Pairwise comparison matrix of F-AHP.

\begin{tabular}{|c|c|c|c|c|c|c|}
\hline & $\mathrm{PT}$ & SC & LA & $\mathrm{AF}$ & GI & $\mathrm{CC}$ \\
\hline PT & $1 / 1 / 1$ & $0.5 / 1 / 1.5$ & $1 / 1.5 / 2$ & $1.5 / 2 / 2.5$ & $1.5 / 2 / 2.5$ & $1 / 1.5 / 2$ \\
\hline SC & $0.67 / 1 / 2$ & $1 / 1 / 1$ & $1 / 1.5 / 2$ & $1.5 / 2 / 2.5$ & $1.5 / 2 / 2.5$ & $1 / 1.5 / 2$ \\
\hline LA & $0.5 / 0.67 / 1$ & $0.5 / 0.67 / 1$ & $1 / 1 / 1$ & $1 / 1.5 / 2$ & $1 / 1.5 / 2$ & $0.5 / 1 / 1.5$ \\
\hline $\mathrm{AF}$ & $0.4 / 0.5 / 0.67$ & $0.4 / 0.5 / 0.67$ & $0.5 / 0.67 / 1$ & $1 / 1 / 1$ & $0.5 / 1 / 1.5$ & $0.5 / 0.67 / 1$ \\
\hline GI & $0.4 / 0.5 / 0.67$ & $0.4 / 0.5 / 0.67$ & $0.5 / 0.67 / 1$ & $0.67 / 1 / 2$ & $1 / 1 / 1$ & $0.5 / 0.67 / 1$ \\
\hline $\mathrm{CC}$ & $0.5 / 0.67 / 1$ & $0.5 / 0.67 / 1$ & $0.67 / 1 / 2$ & $1 / 1.5 / 2$ & $1 / 1.5 / 2$ & $1 / 1 / 1$ \\
\hline
\end{tabular}

TABLE 10: Fuzzy importance ratings of $\mathbf{L}_{1}$ obtained from EA, MEA, and LMM.

\begin{tabular}{lcccccc}
\hline & & & Importance ratings of $\mathbf{L}_{1}$ by different F-AHP & LMM & Equation $(25)$ \\
\hline PT & EA & Equation $(25)$ & MEA & Equation (25) & 0.223 \\
SC & $0.12 / 0.23 / 0.4$ & 0.25 & $0.13 / 0.23 / 0.34$ & 0.23 & $0.21 / 0.23 / 0.23$ & $0.22 / 0.23 / 0.24$ \\
LA & $0.13 / 0.23 / 0.41$ & 0.26 & $0.14 / 0.23 / 0.35$ & 0.24 & $0.14 / 0.15 / / 0.17$ & 0.157 \\
AF & $0.06 / 0.11 / 0.2$ & 0.18 & $0.09 / 0.16 / 0.26$ & 0.17 & $0.11 / 0.11 / 0.12$ & $0.111 / 0.11 / 0.12$ \\
GI & $0.07 / 0.11 / 0.22$ & 0.12 & $0.07 / 0.11 / 0.18$ & 0.12 & 0.114 \\
CC & $0.09 / 0.16 / 0.31$ & 0.13 & $0.07 / 0.11 / 0.20$ & 0.13 & $0.15 / 0.16 / 0.18$ & 0.161 \\
\hline
\end{tabular}

TABLE 11: Comparison analysis between truth and nonspecificity.

\begin{tabular}{lccccccc}
\hline \multirow{2}{*}{$\mathbf{L}_{1}$} & \multicolumn{3}{c}{ Nonspecificity by different F-AHPs } & \multicolumn{2}{c}{$\mathbf{L}_{1}$} & \multicolumn{3}{c}{ Truth of $\mathbf{L}_{1}$ by different F-AHPs } \\
& EA & MEA & LMM & & EA & MEA & LMM \\
\hline PT & 1.5 & 1.11 & $\mathbf{0 . 0 9}$ & PT & $\mathbf{0 . 8 8}$ & 0.80 & 0.5 \\
SC & 1.58 & 1.15 & $\mathbf{0 . 1}$ & SC & $\mathbf{0 . 5 4}$ & 0.45 & 0.25 \\
LA & 1.14 & 0.91 & $\mathbf{0 . 1 1}$ & LA & $\mathbf{0 . 6}$ & 0.51 & 0.30 \\
AF & 0.76 & 0.66 & $\mathbf{0 . 0 4}$ & AF & $\mathbf{0 . 4 4}$ & 0.35 \\
GI & 0.84 & 0.71 & $\mathbf{0 . 0 5}$ & GI & $\mathbf{0 . 4 9}$ & 0.48 & 0.28 \\
CC & 1.22 & 0.96 & $\mathbf{0 . 1 2}$ & CC & $\mathbf{0 . 6 1}$ & 0.48 & 0.5 \\
\hline Max nonspecificity & 1.58 & 1.15 & $\mathbf{0 . 1 8}$ & Min truth & $\mathbf{0 . 4 4}$ & 0.42 & 0.25 \\
\hline
\end{tabular}

TABLE 12: Fuzzy important ratings of some service stations.

\begin{tabular}{ccccccccc}
\hline$\alpha$-cut & Bengbu south & Beijing south & Chengdu & Guangzhou south & Hankou & Nanjing south & Shanghai Hongqiao & Xian north \\
\hline$(\widetilde{A})_{0}^{L}$ & 0.054 & 0.114 & 0.069 & 0.118 & 0.110 & 0.123 & 0.142 & 0.078 \\
$(\widetilde{A})_{1}^{L}$ & 0.060 & 0.152 & 0.081 & 0.152 & 0.136 & 0.152 & 0.173 \\
$(\widetilde{A})_{0}^{U}$ & 0.070 & 0.180 & 0.099 & 0.182 & 0.170 & 0.191 & 0.093 \\
\hline
\end{tabular}


TABLE 13: Relation matrices of FD (between $\mathbf{L}_{2}$ and $\mathbf{L}_{3}$ ).

\begin{tabular}{|c|c|c|c|c|c|c|c|}
\hline & QL & AT & AC & $\mathrm{PC}$ & IR & $\mathrm{F}$ & \\
\hline 1 & $0.006 / 0.007 / 0.007$ & $/ 0.011$ & $66 / 0$. & $07 / 0$ & 69 & 08/0.01 & c \\
\hline 2 & $0.006 / 0.007 / 0.007$ & $0.006 / 0.007 / 0.008$ & $0.006 / 0.007 / 0.008$ & $0.006 / 0.007 / 0.008$ & $7 / 0.008 / 0.009$ & $0.007 / 0.008 / 0.01$ & 08/0.009 \\
\hline 3 & $/ 0.00$ & 08 & $6 / 0.0$ & $6 / 0.0$ & 7/0.008/ & /0.01 & 009 \\
\hline 4 & $0.022 / 0.023 / 0.023$ & /0.011 & $17 / 0$ & 2 & 017 & .023 & 27 \\
\hline 5 & $0.022 / 0.023 / 0.023$ & $0.012 / 0.013 / 0.015$ & $0.018 / 0.019 / 0.02$ & $0.017 / 0.019 / 0.02$ & 0.014/0.016/0.017 & $0.02 / 0.021 / 0.023$ & $0.026 / 0.027 / 0.027$ \\
\hline 6 & $0.022 / 0.023 / 0.023$ & $0.012 / 0.013 / 0.015$ & $0.024 / 0.025 / 0.025$ & $0.024 / 0.025 / 0.025$ & $/ 0.016 / 0.017$ & .023 & .027 \\
\hline 7 & 0.0 & $0.006 / 0.00$ & $0.006 / 0.007 / 0.008$ & $0.006 / 0.007 / 0$ & $0.007 / 0.008 / 0.009$ & $0.007 / 0$ & .009 \\
\hline 8 & $0.006 / 0.007 / 0.007$ & 0.008/0.009/0.011 & $0.006 / 0.007 / 0.008$ & $0.006 / 0.007 / 0.008$ & $0.007 / 0.008 / 0.009$ & $0.007 / 0.008 / 0.01$ & $0.007 / 0.008 / 0.009$ \\
\hline & $0.008 / 0.0$ & $0.012 / 0.0$ & $0.009 /($ & 16 & 0.00 & .013 & 13 \\
\hline 10 & $0.008 / 0$. & $0.012 / 0$ & 011 & 16 & 0. & & \\
\hline 11 & $0.011 / 0.013 / 0.014$ & $0.008 / 0.009 / 0.011$ & $0.012 / 0.014 / 0.015$ & 0.009/0.01/0.011 & $01 / 0.011 / 0.013$ & $0.007 / 0.008 / 0.01$ & $0.007 / 0.008 / 0.009$ \\
\hline 2 & $0.008 / 0.009 / 0.01$ & $0.008 / 0.0$ & $0.009 / 0.0$ & $0.012 / 0.014 / 0$ & $1 / 0.011 / 0.013$ & $8 / 0.01$ & .01 \\
\hline 13 & $0.006 / 0.007 / 0.007$ & $0.006 / 0.007 / 0.008$ & $0.006 / 0.0 \mathrm{C}$ & /0.007/0 & 0.06 & 0. & 0.01 \\
\hline 14 & $/ 0.013 / 0.014$ & $012 / 0.0$ & $0.009 / 0$ & $0.012 / 0.014 / 0.016$ & $/ 0.00$ & 0. & 01 \\
\hline 15 & /0.01 & $0.012 / 0$. & $0.009 / 0$ & 16 & 13 & .01 & \\
\hline 16 & $0.022 / 0.023 / 0.023$ & $0.017 / 0.0$ & $0.012 / 0$ & $0.012 / 0$ & $0.021 / 0.0$ & 0.0 & 022 \\
\hline 17 & $0.022 / 0.023 / 0.023$ & $0.017 / 0.0$ & 0.012 & 16 & 0 & 18 & \\
\hline 18 & $0.022 / 0.023 / 0.023$ & $0.023 / 0.0$ & $0.018 / 0$. & 0.01 & $0 .($ & 23 & \\
\hline 9 & .01 & 011 & 08 & 0. & 13 & & \\
\hline 20 & $8 / 0$. & $0.008 / 0.0$ & $0.012 / 0 . c$ & 0.0 & 17 & 014 & ${ }_{1}$ \\
\hline 21 & $0.006 / 0.007 / 0.007$ & $0.006 / 0.007 / 0.008$ & $0.006 / 0.007 / 0.008$ & $0.006 / 0.007 / 0.008$ & $0.01 / 0.011 / 0.013$ & $0.007 / 0.008 / 0.01$ & $8 / 0.01$ \\
\hline 22 & $0.016 / 0.017 / 0.018$ & $0.023 / 0.024 / 0.024$ & $0.018 / 0.019 / 0.02$ & $0.018 / 0$. & $0.014 / 0.016 / 0.017$ & $5 / 0.018$ & $6 / 0.017$ \\
\hline 23 & 0.016/0.017/0.018 & $017 / 0.0$ & $024 / 0.0$ & 0. & 0. & 0. & 0.01 \\
\hline 24 & $0.016 / 0.017 / 0.018$ & $0.017 / 0.0$ & $0.024 / 0.025 / 0.025$ & 02 & /0.016/0.017 & 0.023 & 017 \\
\hline 25 & $0.011 / 0.013 / 0.014$ & $0.012 / 0$ & $0.018 / 0$. & 0.01 & $0 .($ & 0.0 & 0.0 \\
\hline 26 & $0.006 / 0.007 / 0.007$ & $0.006 / 0.007 / 0.008$ & $0.006 / 0.007 / 0.008$ & $0.006 / 0$. & $0.007 / 0.0$ & .01 & 0. \\
\hline 27 & $0.006 / 0.007 / 0.008$ & $0.006 / 0.007 / 0.008$ & $0.006 / 0.007 / 0.008$ & $0.006 / 0.007 / 0.008$ & $0.007 / 0.008 / 0$ & 0.01 & .01 \\
\hline 28 & $0.008 / 0.009 / 0.01$ & $0.017 / 0.0$ & $0.012 / 0$ & 16 & 0.00 & 0. & $0 .($ \\
\hline 29 & $0.008 / 0.0$ & $0.017 /$ & $0.012 /$ & 0.0 & 0.00 & 0. & 01 \\
\hline 30 & $0.006 / 0.007 / 0.008$ & $0.006 / 0.00$ & $0.006 / 0.007 / 0.008$ & $0.006 / 0.007 / 0.008$ & $1 / 0.011 / 0.013$ & $8 / 0.01$ & 08/0.01 \\
\hline 31 & $0.006 / 0.007 / 0$. & $0.006 / 0.007 / 0.008$ & $0.007 / 0.007 / 0.009$ & $0.006 / 0.007 / 0$. & $0.007 / 0.008 / 0.009$ & $0.007 / 0.008 / 0.01$ & $0.007 / 0.008 / 0.01$ \\
\hline 32 & $0.006 / 0.007 / 0.008$ & $0.006 / 0.007 / 0.008$ & $0.009 / 0.01 / 0.012$ & $0.006 / 0.007 / 0.008$ & $0.007 / 0.008 / 0.009$ & $0.007 / 0.008 / 0.01$ & $0.007 / 0.008 /$ \\
\hline 33 & $0.006 / 0.007 / 0.008$ & 0.008/0.009/0.011 & $0.009 / 0.01 / 0.012$ & $0.006 / 0.007 / 0.008$ & $0.007 / 0.008 / 0.009$ & $0.007 / 0.008 / 0.01$ & $0.009 / 0.011 / 0.013$ \\
\hline 34 & $0.006 / 0.007 / 0.008$ & $0.006 / 0.007 / 0.008$ & $0.007 / 0.0$ & $0.007 / 0.007 / 0$ & $0.007 / 0.0$ & $0.007 / 0$ & $0.007 / 0.008 / 0.01$ \\
\hline 35 & $0.006 / 0.007 / 0.008$ & $0.006 / 0.007 / 0.008$ & $0.007 / 0.007 / 0.009$ & $0.007 / 0.007 / 0.009$ & $0.01 / 0.011 / 0.013$ & 08/0.01 & $0.007 / 0$ \\
\hline 36 & $0.006 / 0.007 / 0.008$ & $0.006 / 0.007 / 0.008$ & $0.007 / 0.007 / 0.009$ & $0.007 / 0.007 / 0.009$ & $0.007 / 0.008 / 0.009$ & $0.007 / 0.008 / 0.01$ & $0.007 / 0.008 / 0.01$ \\
\hline 37 & $0.006 / 0.007 / 0$ & $0.006 / 0$. & $0.007 / 0.007 / 0.009$ & $0.007 / 0$ & $0.007 / 0.008 / 0$ & $0.007 / 0.008 / 0.01$ & $.007 / 0.008 / 0.01$ \\
\hline 38 & $0.006 / 0.007 / 0.008$ & $0.006 / 0.007 / 0.008$ & $0.007 / 0.007 / 0.009$ & $0.007 / 0.007 / 0.009$ & $0.007 / 0.008 / 0.009$ & $0.007 / 0.008 / 0.01$ & $0.007 / 0.008 / 0.01$ \\
\hline 39 & $/ 0.013 / 0.014$ & 0.008/0.009/0.011 & $0.012 / 0.014 / 0.016$ & 4/0.016 & $0.014 / 0.016 / 0.018$ & $1 / 0.014$ & $0.014 / 0.016 / 0.018$ \\
\hline 40 & $0.011 / 0.013 / 0.014$ & $0.012 / 0.013 / 0.015$ & $0.009 / 0.01 / 0.012$ & $0.012 / 0.014 / 0.016$ & $0.014 / 0.016 / 0.018$ & $0.014 / 0.016 / 0.018$ & $0.009 / 0.011 / 0.013$ \\
\hline 41 & $0.016 / 0.017 / 0.018$ & $0.023 / 0.024 / 0.024$ & $0.013 / 0.014 / 0.016$ & $0.012 / 0.014 / 0.016$ & $0.014 / 0.016 / 0.018$ & 0.01/0.011/0.014 & $0.009 / 0.011 / 0.013$ \\
\hline 42 & $0.016 / 0.017 / 0.018$ & $0.023 / 0.024 / 0.024$ & $0.018 / 0.019 / 0.02$ & $0.024 / 0.025 / 0.025$ & 0. & /0.023 & 0.0 \\
\hline 43 & $0.011 / 0.013 / 0.014$ & $0.012 / 0.013 / 0.015$ & $0.009 / 0.01 / 0.012$ & $0.012 / 0.014 / 0.016$ & $0.014 / 0.016 / 0.018$ & $0.007 / 0.008 / 0.01$ & $0.009 / 0.011 / 0.013$ \\
\hline 44 & $0.016 / 0.017 / 0.019$ & $0.012 / 0.013 / 0.015$ & $0.009 / 0.01 / 0.012$ & $0.009 / 0.01 / 0.011$ & $0.01 / 0.011 / 0.013$ & $0.007 / 0.008 / 0.01$ & $0.009 / 0.011 / 0.013$ \\
\hline 45 & $0.008 / 0.009 / 0.01$ & $0.006 / 0.007 / 0.008$ & $0.007 / 0.007 / 0.009$ & 0.0 & 13 & 014 & $0.01 / 0.011 / 0.013$ \\
\hline 46 & $0.011 / 0.013 / 0.014$ & $0.012 / 0.6$ & $0.007 / 0.007 / 0.0$ & 09 & $0.007 / 0$. & $/ 0.014$ & $0.014 / 0.016 / 0.018$ \\
\hline 47 & $0.012 / 0.013 / 0.014$ & $0.012 / 0.013 / 0.015$ & $0.007 / 0.007 / 0.009$ & $0.007 / 0.007 / 0.009$ & $0.007 / 0.008 / 0.01$ & $0.01 / 0.011 / 0.014$ & $0.014 / 0.016 / 0.018$ \\
\hline
\end{tabular}


TABLE 13: Continued.

\begin{tabular}{|c|c|c|c|c|c|c|c|}
\hline & QL & $\mathrm{TT}$ & $\mathrm{C}$ & PC & HR & $\mathrm{EF}$ & PI \\
\hline 48 & 000 & 8 & 09 & 09 & $7 / 0.008 / 0.01$ & $/ 0.0$ & 10.0 \\
\hline ty & 9 & & & mets & & & \\
\hline DO & 1 & 06 & $0.007 / 0.007$ & $0.007 / 0.007 / 0.009$ & & & \\
\hline 51 & $0.022 / 0.023 / 0.023$ & $23 / 0.024 / 0.024$ & $0.018 / 0.019 / 0.021$ & 018/0.019/0.021 & $14 / 0.016 / 0.018$ & 01/0.011/0.014 & \\
\hline 52 & $0.022 / 0.023 / 0.023$ & $0.017 / 0.01$ & 25 & 25 & & 23 & 027 \\
\hline 53 & $0.016 / 0.017 / 0.019$ & $0.012 / 0.013 / 0.015$ & $0.018 / 0.019 / 0.021$ & $018 / 0019 / 0,021$ & $14 / 0.016 / 0.018$ & 014/0.016/0.018 & \\
\hline & & & & & $0.021 / 0.022 / 0.022$ & 018 & \\
\hline & & & & & & & \\
\hline & $/ 0.00$ & 11 & & & & & \\
\hline (7) & $0.006 / 0.007 /($ & $0.006 / 0.007 / 0.008$ & $0.007 / 0.007 / 0.009$ & $0.007 / 0.007 / 0.009$ & $.007 / 0.008 / 0.01$ & 07/0.008/0.011 & 0.01 \\
\hline 58 & 11 & 1 & 6 & 17 & 13 & 11 & 01 \\
\hline 59 & 11 & 0. & 6 & 12 & 18 & & \\
\hline 60 & 1 & 0.01 & 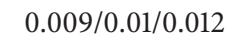 & 12100 & & & \\
\hline 61 & & & & & & & \\
\hline 62 & & & & & & & \\
\hline 63 & & & & & & & \\
\hline 64 & $0.013 / 0.014$ & 0.011 & & & & & \\
\hline 65 & /0.017/0.019 & $0.009 / 0.009 / 0.011$ & & 12 & & & \\
\hline 56 & $0.017 / 0.019$ & $023 / 0.024 / 0.024$ & 018/0.019/0.021 & 0.021 & 018 & 14 & .018 \\
\hline . & 19 & 4 & 21 & /0.019/0.021 & .019 & 14 & .018 \\
\hline 68 & 1001210 & $017 / 0018 / 002$ & $0012 / 001410$ & $3 / 0.014 / 0.017$ & $.01 / 0.011 / 0.013$ & & \\
\hline 69 & & & & & & & \\
\hline 70 & 8 & & & & & & \\
\hline 71 & & & & & & & \\
\hline 72 & & & & & & & \\
\hline 73 & & & & & & & 011 \\
\hline & $06 / 0.007 / 0.008$ & $0.007 / 0$ & 0.0 & 99 & 01 & & \\
\hline & $006 / 0.007 / 0.008$ & $0.009 / 0.009 / 0.012$ & (1) & 促 & .01 & 011 & \\
\hline & & & & & & & \\
\hline 77 & & & & & & & \\
\hline 78 & $0.006 / 0.007 / 0.008$ & $0.007 / 0.007 / 0.008$ & $0.007 / 0.007 / 0.009$ & $0.007 / 0.007 / 0.009$ & & & \\
\hline 79 & 15 & & & & & & \\
\hline 80 & $0.012 / 0.013 / 0.015$ & $0.009 / 0.009 / 0.012$ & & $09 / 0.01 / 0.012$ & & & $0.01 / 0.011 / 0.014$ \\
\hline 81 & & & & & & & \\
\hline 82 & & & & & & & \\
\hline 02 & & & & & & & \\
\hline 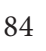 & & & & & & & \\
\hline 85 & $0.012 / 0.013 / 0.015$ & $.009 / 0.009 / 0.012$ & $0.009 / 0.01 / 0.012$ & $0.009 / 0.01 / 0.012$ & $0.01 / 0.011 / 0.014$ & $0.01 / 0.011 / 0.015$ & $0.01 / 0.011 / 0.014$ \\
\hline
\end{tabular}

$a / b / c$; the maximum of a box is $c$ in fuzzy triangle number $a / b / c)$. Based on LMM inputs and the approximate reasoning procedure in SSEP-CSHR, we know that the outputs only have truth value 0.25 (see Table 11).

\section{Conclusions}

QFD is a method to specify the customer wants and needs and then to evaluate each alternative in terms of its impact on meeting those needs. This paper is an effort to improve the scientific credibility and practicability of F-QFD and to offer a qualitative approach for SSEP-CSHR based on the two-phase F-QFD with an approximate procedure based on evidence theory. Belief and Plausibility are two types of measures in the view of evidence theory. Using the two measures, a framework to determine the truth of " $x$ is $\mathbf{A}$ " is investigated in Section 2 and then is used to deal with different types of BOE that might be met in CSHR's survey. The contribution of Section 3 is twofold. Firstly, this paper offers a MCDM 
approach for SSEP-CSHR based on the two-phase F-QFD to select potential service stations for CSHR with regard to series of practical criteria and Basic Requirements in context of CSHR. Secondly, this paper illustrates that the usage of evidence theory allows an approximate reasoning based on various types of $\mathrm{BOE}$ to be incorporated into F-QFD. In our case study, the proposed method is implemented in a high-speed rail network of China referring to 85 railway stations. The application, on the one hand, presents the fuzzy importance ratings for 85 service stations; on the other hand, it shows the truth of our Conclusions to be assessed by using proposed evidence-based inference schema. It demonstrated that the proposed method enhances scientific credibility of F-QFD in SSEP-CSHR and allows decision makers to express how much they know. By deep analysis, we realize an interesting trade-off between nonspecificity and truth in F-QFD. Hence our experiments partly justify a diversity of "uncertainty management."

\section{Appendix}

See Table 13.

\section{Conflict of Interests}

The authors declare that there is no conflict of interests regarding the publication of this paper.

\section{Acknowledgments}

This research was supported by the following funds: evaluation and system research about line planning of highspeed railway (China Railway Corporation) (Grant no. 2014X010-A); National Natural Science Foundation of China (U1434207); Fundamental Research Funds for the Central Universities (Beijing Jiaotong University) (Grant no. 2014JBZ008).

\section{References}

[1] R. J. Kuo, S. C. Chi, and S. S. Kao, "A decision support system for locating convenience store through fuzzy AHP," Computers \& Industrial Engineering, vol. 37, no. 1, pp. 323-326, 1999.

[2] C.-T. Chen, "A fuzzy approach to select the location of the distribution center," Fuzzy Sets and Systems, vol. 118, no. 1, pp. 65-73, 2001.

[3] C. Kahraman, D. Ruan, and I. Doğan, "Fuzzy group decisionmaking for facility location selection," Information Sciences, vol. 157, no. 1-4, pp. 135-153, 2003.

[4] J. M. Blin, "Fuzzy relations in group decision theory," Journal of Cybernetics, vol. 4, pp. 17-22, 1974.

[5] R. R. Yager, "Fuzzy decision-making including unequal objectives," Fuzzy Sets and Systems, vol. 1, no. 2, pp. 87-95, 1978.

[6] D.-Y. Chang, "Applications of the extent analysis method on fuzzy AHP," European Journal of Operational Research, vol. 95, no. 3, pp. 649-655, 1996.

[7] O. Kulak and C. Kahraman, "Fuzzy multi-attribute selection among transportation companies using axiomatic design and analytic hierarchy process," Information Sciences, vol. 170, no. 2-4, pp. 191-210, 2005.

[8] P. J. Guo, "Fuzzy data envelopment analysis and its application to location problems," Information Sciences, vol. 179, no. 6, pp. 820-829, 2009.

[9] Y. Akao, "QFD: past, present and future," in Proceedings of the International Symposium on QFD, pp. 1-12, Linköping, Sweden, October 1997.

[10] G. H. Mazur, "QFD for service industries-from voice of customer to task deployment," in Proceedings of the 5th Symposium on Quality Function Deployment, pp. 1-17, Novi, Mich, USA, June 1993.

[11] A. Jamalnia, H. A. Mahdiraji, M. R. Sadeghi, S. H. R. Hajiagha, and A. Feili, "An integrated fuzzy QFD and fuzzy goal programming approach for global facility location-allocation problem," International Journal of Information Technology \& Decision Making, vol. 13, no. 2, pp. 263-290, 2014.

[12] S. Kikuchi and P. Chakroborty, "Place of possibility theory in transportation analysis," Transportation Research Part B: Methodological, vol. 40, no. 8, pp. 595-615, 2006.

[13] K.-J. Kim, D.-H. Kim, and D.-K. Min, "Robust QFD: framework and a case study," Quality and Reliability Engineering International, vol. 23, no. 1, pp. 31-44, 2007.

[14] C. Temponi, J. Yen, and W. A. Tiao, "House of quality: a fuzzy logic-based requirements analysis," European Journal of Operational Research, vol. 117, no. 2, pp. 340-354, 1999.

[15] C. K. Kwong and H. Bai, "Determing the importance weights for the customer requirements in QFD using a fuzzy AHP with an extent analysis approach," IIE Transactions, vol. 35, pp. 619-626, 2003.

[16] C. Kahraman, T. Ertay, and G. Büyüközkan, "A fuzzy optimization model for QFD planning process using analytic network approach," European Journal of Operational Research, vol. 171, no. 2, pp. 390-411, 2006.

[17] L.-H. Chen and W.-C. Ko, "Fuzzy linear programming models for new product design using QFD with FMEA," Applied Mathematical Modelling, vol. 33, no. 2, pp. 633-647, 2009.

[18] L.-H. Chen and W.-C. Ko, "Fuzzy linear programming models for NPD using a four-phase QFD activity process based on the means-end chain concept," European Journal of Operational Research, vol. 201, no. 2, pp. 619-632, 2010.

[19] E. Bottani and A. Rizzi, "Strategic management of logistics service: a fuzzy QFD approach," International Journal of Production Economics, vol. 103, no. 2, pp. 585-599, 2006.

[20] Y.-M. Wang and K.-S. Chin, "Technical importance ratings in fuzzy QFD by integrating fuzzy normalization and fuzzy weighted average," Computers \& Mathematics with Applications, vol. 62, no. 11, pp. 4207-4221, 2011.

[21] T. L. Saaty, "A scaling method for priorities in hierarchical structures," Journal of Mathematical Psychology, vol. 15, no. 3, pp. 234-281, 1977.

[22] J. J. Buckley, T. Feuring, and Y. Hayashi, "Fuzzy hierarchical analysis," in Proceedings of the IEEE International Fuzzy Systems Conference Proceedings (FUZZ-IEEE '99), vol. 2, pp. 1009-1013, IEEE, Seoul, Republic of Korea, August 1999.

[23] K.-J. Zhu, Y. Jing, and D.-Y. Chang, "Discussion on extent analysis method and applications of fuzzy AHP," European Journal of Operational Research, vol. 116, no. 2, pp. 450-456, 1999.

[24] Y.-M. Wang, Y. Luo, and Z. Hua, "On the extent analysis method for fuzzy AHP and its applications," European Journal of Operational Research, vol. 186, no. 2, pp. 735-747, 2008. 
[25] R. Csutora and J. J. Buckley, "Fuzzy hierarchical analysis: the Lambda-Max method," Fuzzy Sets and Systems, vol. 120, no. 2, pp. 181-195, 2001.

[26] D.-H. Kim and K.-J. Kim, "Robustness indices and robust prioritization in QFD," Expert Systems with Applications, vol. 36, no. 2, pp. 2651-2658, 2009.

[27] G. J. Klir and B. Yuan, Fuzzy Sets and Fuzzy Logic-Theory and Application, Prentice Hall PTR, Upper Saddle River, NJ, USA, 1995.

[28] Y.-M. Wang and T. M. Elhag, "On the normalization of interval and fuzzy weights," Fuzzy Sets and Systems, vol. 157, no. 18, pp. 2456-2471, 2006.

[29] Y.-M. Wang, "Centroid defuzzification and the maximizing set and minimizing set ranking based on alpha level sets," Computers \& Industrial Engineering, vol. 57, no. 1, pp. 228-236, 2009.

[30] L. A. Zadeh, "Outline of a new approach to the analysis of complex systems and decision processes," IEEE Transactions on Systems, Man and Cybernetics, vol. 3, no. 1, pp. 28-44, 1973. 


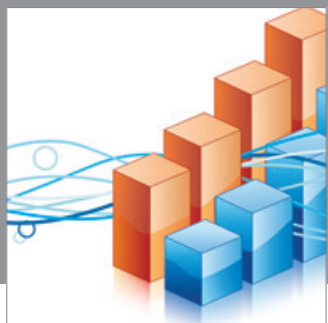

Advances in

Operations Research

mansans

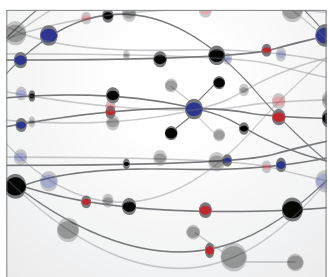

The Scientific World Journal
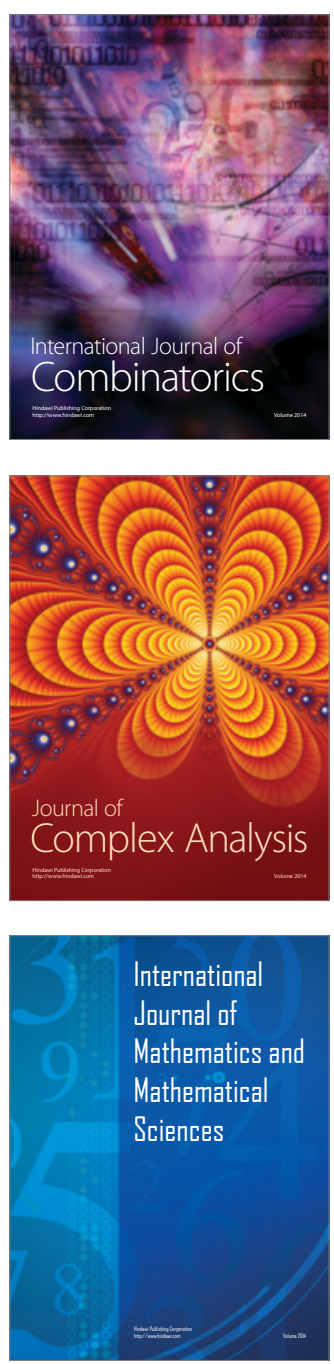
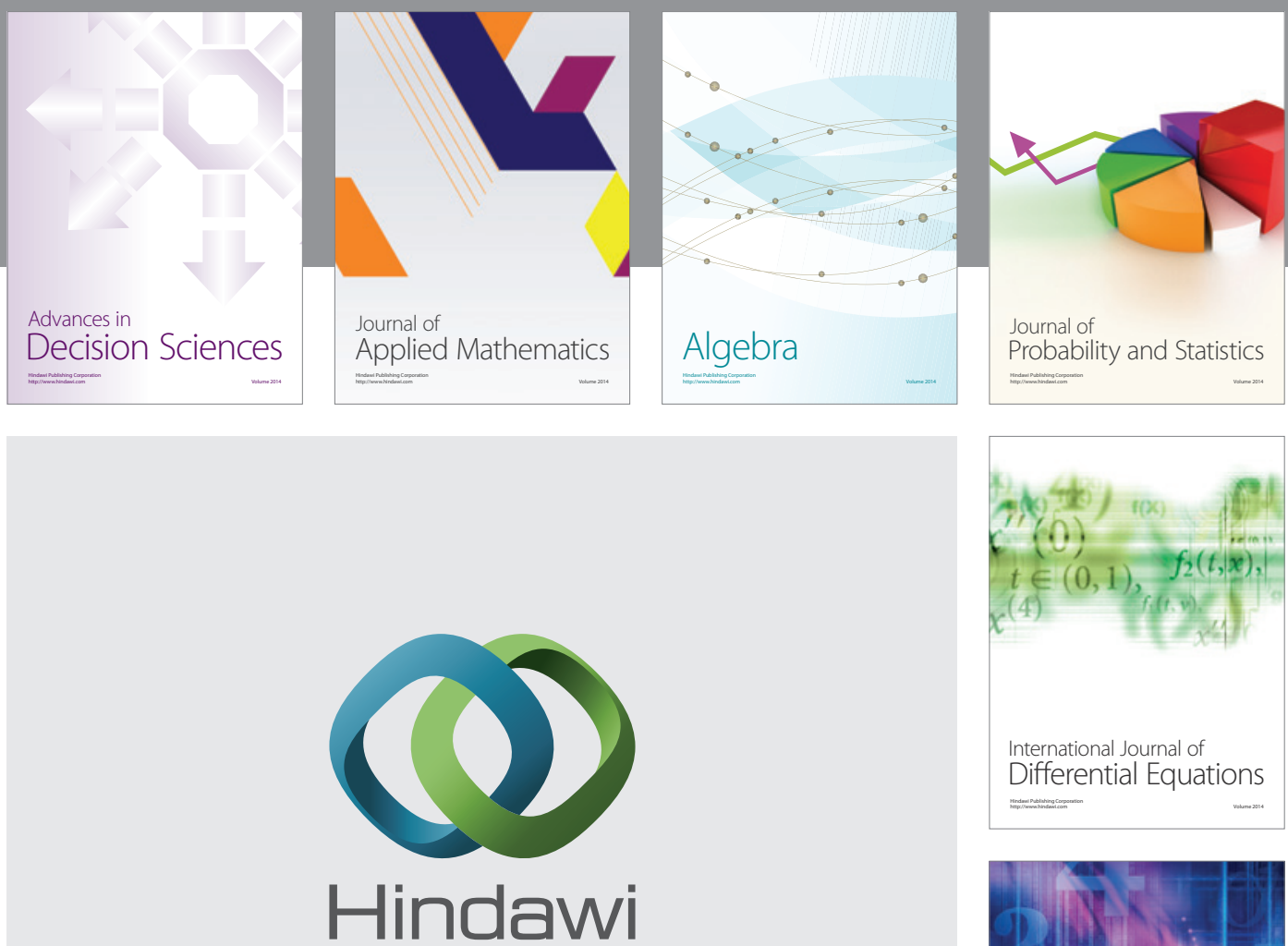

Submit your manuscripts at http://www.hindawi.com
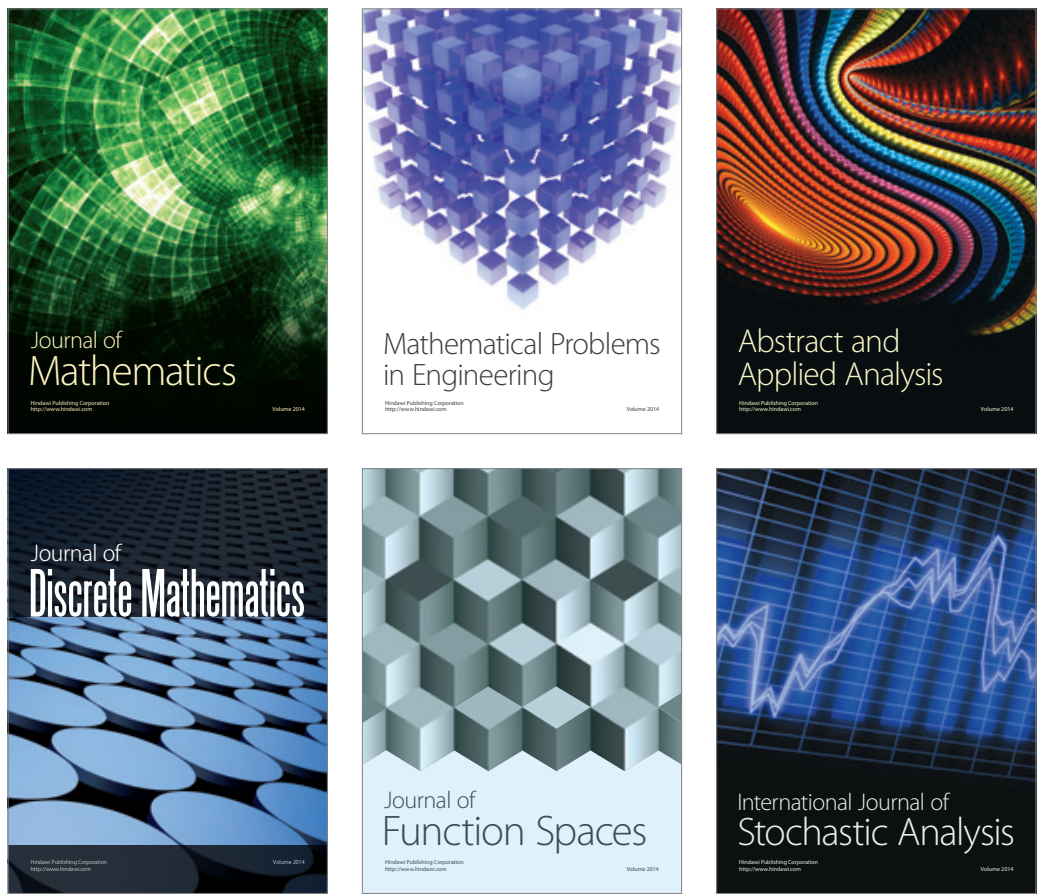

Journal of

Function Spaces

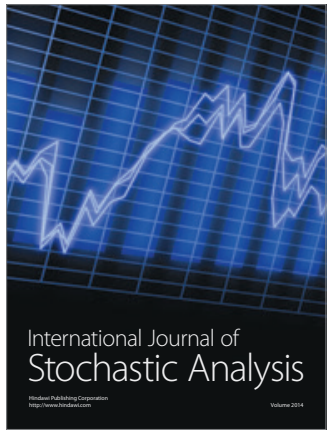

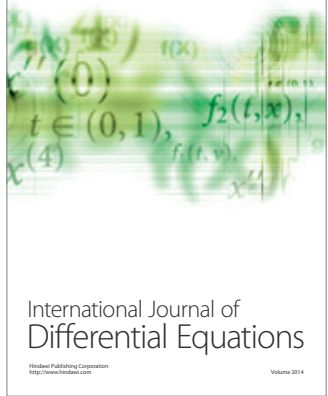
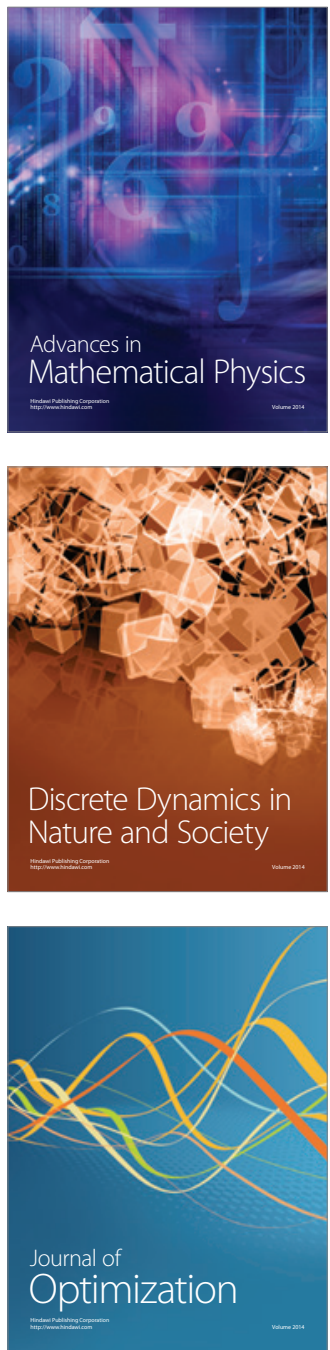